\title{
An Efficient Bayesian PAPR Reduction Method for OFDM-Based Massive MIMO Systems
}

\author{
Hengyao Bao, Jun Fang, Zhi Chen, Hongbin Li, Senior Member, IEEE, and Shaoqian Li, Fellow, IEEE
}

\begin{abstract}
We consider the problem of peak-to-average power ratio (PAPR) reduction in orthogonal frequency-division multiplexing (OFDM) based massive multiple-input multiple-output (MIMO) downlink systems. Specifically, given a set of symbol vectors to be transmitted to $K$ users, the problem is to find an OFDM-modulated signal that has a low PAPR and meanwhile enables multiuser interference (MUI) cancelation. Unlike previous works that tackled the problem using convex optimization, we take a Bayesian approach and develop an efficient PAPR reduction method by exploiting the redundant degrees-of- freedom of the transmit array. The sought-after signal is treated as a random vector with a hierarchical truncated Gaussian mixture prior, which has the potential to encourage a low PAPR signal with most of its samples concentrated on the boundaries. A variational expectation-maximization (EM) strategy is developed to obtain estimates of the hyperparameters associated with the prior model, along with the signal. In addition, the generalized approximate message passing (GAMP) is embedded into the variational EM framework, which results in a significant reduction in computational complexity of the proposed algorithm. Simulation results show our proposed algorithm achieves a substantial performance improvement over existing methods in terms of both the PAPR reduction and computational complexity.
\end{abstract}

Index Terms-Massive MIMO-OFDM, PAPR reduction, variational EM, GAMP.

\section{INTRODUCTION}

Massive multiple-input multiple-output (MIMO), also known as large-scale or very-large MIMO, is a promising technology to meet the ever growing demands for higher throughput and better quality-of-service of next-generation wireless communication systems [1]. Massive MIMO systems are those that are equipped with a large number of antennas at the base station (BS) simultaneously serving a much smaller number of single-antenna users sharing the same time-frequency bandwidth. In addition to higher throughput, massive MIMO systems also have the potential to improve the energy efficiency and enable the use of inexpensive, lowpower components. Hence, it is expected that massive MIMO will bring radical changes to future wireless communication systems.

In practice, broadband wireless communications may suffer from frequency-selective fading. Orthogonal frequencydivision multiplexing (OFDM), a scheme of encoding digital

Hengyao Bao, Jun Fang, Zhi Chen, and Shaoqian Li are with the National Key Laboratory of Science and Technology on Communications, University of Electronic Science and Technology of China, Chengdu 611731, China, Email: JunFang@uestc.edu.cn, chenzhi@uestc.edu.cn, 1sq@uestc.edu.cn

Hongbin $\mathrm{Li}$ is with the Department of Electrical and Computer Engineering, Stevens Institute of Technology, Hoboken, NJ 07030, USA, E-mail: Hongbin.Li@stevens.edu

This work was supported in part by the National Science Foundation of China under Grant 61172114. data on multiple carrier frequencies, has been widely used to deal with frequency-selective fading. However, a major problem associated with the OFDM is that it is subject to a high peak-to-average power ratio (PAPR) owing to the independent phases of the sub-carriers [2]. To avoid outof-band radiation and signal distortion, handling this high PAPR requires a high-resolution digital-to-analog converter (DAC) and a linear power amplifier (PA) at the transmitter, which is not only expensive but also power-inefficient [3]. The situation deteriorates when the number of antennas is large, leaving such systems impractical. Therefore, it is of crucial importance to reduce the PAPR of massive MIMO-OFDM systems to facilitate low-cost and power-efficient hardware implementations.

Many techniques have been developed for PAPR reduction in single-input single-output (SISO) OFDM wireless systems. The most prominent are clipping [4], tone reservation (TR) [5], active constellation extension (ACE) [6], selected mapping (SLM) [7], partial transmission sequence (PTS) [8] and others. For a detailed overview, we refer readers to [3], [9]. Although these PAPR-reduction schemes can be extended to point-topoint MIMO systems easily [9]-[11], extension to the multiuser (MU) MIMO downlink is not straightforward, mainly because joint receiver-side signal processing is almost impossible in practice as the users are distributed. Recently, a new PAPR reduction method [12] was developed for massive MIMOOFDM systems. The proposed scheme utilizes the redundant degrees-of-freedom (DoFs) resulting from the large number of antennas at the BS to achieve joint multiuser interference (MUI) cancelation and PAPR reduction. Specifically, the problem was formulated as a linear constrained $\ell_{\infty}$ optimization problem and a fast iterative truncation algorithm (FITRA) was developed in [12]. However, the FITRA algorithm shows to have a fairly low convergence rate. Also, the algorithm employs a regularization parameter to achieve balance between the PAPR reduction and the MUI cancelation (i.e. data fitting error). The choice of the regularization parameter may be tricky in practice. On the other hand, the regularization parameter may be seen instead as an additional degree of freedom that allows to regulate the operation of the algorithm. In [13], a peak signal clipping scheme was employed to reduce the PAPR and some of the antennas at the BS are reserved to compensate for peak-clipping signals. This method has a lower computational complexity. But it achieves only a mild PAPR reduction and those antennas reserved for compensation may incur large PAPRs.

In this paper, we develop a novel Bayesian approach to address the joint PAPR reduction and MUI cancelation prob- 
lem for downlink multi-user massive MIMO-OFDM systems. Specifically, MUI cancelation can be formulated as an underdetermined linear inverse problem which admits numerous solutions. To search for a low PAPR solution, a hierarchical truncated Gaussian mixture prior model is proposed and assigned to the unknown signal (i.e. solution). This hierarchical prior has the potential to encourage a quasi-constant magnitude solution with as many entries as possible lying on the truncated boundaries, thus resulting in a low PAPR. A variational expectation-maximization (EM) algorithm is developed to obtain estimates of the hyperparameters associated with the prior model, along with the signal. In addition, the generalized approximate message passing (GAMP) technique [14] is employed to facilitate the algorithm development in the expectation step. This GAMP technique also helps significantly reduce the computational complexity of the proposed algorithm. Simulation results show that the proposed method presents a substantial improvement over the FITRA algorithm in terms of both PAPR reduction and computational complexity.

During the review process of the current work, it was brought to our attention that an efficient approximate message passing (AMP)-based Bayesian method was recently proposed [15] for PAPR reduction for massive MIMO systems, which can be extended to the case with OFDM modulation. The rationale behind our work and the above work are similar: both methods cast the PAPR reduction problem as a Bayesian inference problem and employ priors to promote solutions with constant envelopes. The prior distributions employed by these two works, however, are very different. The prior proposed in [15] assigns each coefficient to a random point on a circle with a certain radius on the complex plane. Unlike our work, this prior only encourages entries of the obtained solution to be close to the boundary but cannot guarantee that they exactly lie on the boundary points.

The rest of this paper is organized as follows. In Section we introduce the data model, basic assumptions, and the PAPR reduction problem. A new hierarchical Bayesian prior model is proposed in Section III, and an efficient Bayesian algorithm is developed in Section [V] Simulation results are provided in Section V, followed by concluding remarks in Section VI

Notations: Lowercase boldface is used for column vectors $\boldsymbol{x}$, and uppercase for matrices $\boldsymbol{X}$. The superscripts $(\cdot)^{T}$ and $(\cdot)^{H}$ represent the transpose and conjugate transpose, respectively. $\|\boldsymbol{x}\|_{2}$ is used to denote the $\ell_{2}$ norm of vector $\boldsymbol{x}$, and $\|\boldsymbol{x}\|_{\infty}$ stands for the $\ell_{\infty}$ norm, $\ell_{\widetilde{\infty}}$ norm is define as $\|\boldsymbol{x}\|_{\ell_{\widetilde{\infty}}}=\max \left\{\|\Re\{\boldsymbol{x}\}\|_{\infty},\|\Im\{\boldsymbol{x}\}\|_{\infty}\right\}$, with $\Re\{\boldsymbol{x}\}$ and $\Im\{\boldsymbol{x}\}$ denoting the real and imaginary part of $\boldsymbol{x}$, respectively. $\boldsymbol{F}_{N}$ denotes the $N \times N$ unitary discrete Fourier transform (DFT) matrix. The $N \times N$ identity matrix and the $M \times N$ allzeros matrix are denoted by $\boldsymbol{I}_{N}$ and $\mathbf{0}_{M \times N}$, respectively. We denote the pdf of Gaussian random variable $x$ with mean $\mu$ and variance $\sigma^{2}$ as $\mathcal{N}\left(x ; \mu, \sigma^{2}\right)$, for the special case of $\mathcal{N}(x ; 0,1)$, we write the cdf as $\Phi(x)$. The symbol $\otimes$ denotes the Kronecker product.

\section{System Model And Problem Formulation}

We first introduce the system model of OFDM based massive MIMO systems. Then we discuss some recent research on PAPR reduction for multi-user massive MIMO-OFDM systems.

\section{A. System Model}

The system model of the OFDM-based massive MIMO downlink scenario is depicted in Fig. 11 where the BS is assumed to have $M$ transmit antennas and serve $K$ independent single-antenna users $(K \ll M)$, and the total number of OFDM tones is $N$. In practice, the set of tones available are divided into two sets $\mathcal{T}$ and $\mathcal{T}^{\mathcal{C}}$, where the tones in set $\mathcal{T}$ are used for data transmission and the tones in its complementary set $\mathcal{T}^{\mathcal{C}}$ are used for guard band (unused tones at both ends of the spectrum). Hence, for each tone $n \in \mathcal{T}$, the corresponding $K \times 1$ vector $\boldsymbol{s}_{n}$ comprises the symbols for $K$ users, which are usually chosen from a complex-valued signal alphabet $\mathcal{B}$. We normalize the data vector to satisfy $\mathbb{E}\left\{\left\|\boldsymbol{s}_{n}\right\|_{2}^{2}\right\}=1$. For each tone $n \in \mathcal{T}^{\mathcal{C}}$, we set $\boldsymbol{s}_{n}=\mathbf{0}_{K \times 1}$ such that no signal is transmitted in the guard band.

Since cooperative detection among users is often impossible, precoding must be performed at the BS to remove multiuser interference (MUI). Usually, the signal vector on the $n$th tone is linearly precoded as

$$
\boldsymbol{w}_{n}=\boldsymbol{P}_{n} \boldsymbol{s}_{n}
$$

where $\boldsymbol{w}_{n} \in \mathbb{C}^{M \times 1}$ is the precoded vector that contains symbols to be transmitted on the $n$th sub-carrier through the $M$ antennas respectively, and $\boldsymbol{P}_{n} \in \mathbb{C}^{M \times K}$ represents the precoding matrix for the $n$th OFDM tone. Zero-forcing (ZF) precoding and minimum-mean square-error (MMSE) precoding are two classical precoding schemes. The former aims at removing MUI completely, while the latter tries to achieve balance between the MUI cancellation and the noise enhancement. In this paper, we consider the $\mathrm{ZF}$ procoding scheme. Note that since $K \ll M$, the $\mathrm{ZF}$ precoding matrix has an infinite number of forms, among which the most widely used is

$$
\boldsymbol{P}_{n}^{\mathrm{zf}}=\boldsymbol{H}_{n}^{H}\left(\boldsymbol{H}_{n} \boldsymbol{H}_{n}^{H}\right)^{-1},
$$

where $\boldsymbol{H}_{n} \in \mathbb{C}^{K \times M}$ denotes the MIMO channel matrix associated with the $n$th tone. Here we assume the channel matrix $\boldsymbol{H}_{n}, \forall n$ to be known at the transmitter, which can be acquired by exploiting the channel reciprocity of time division duplexing (TDD) systems (i.e., the downlink channel is the transpose of the uplink channel).

After precoding, all precoded vectors $\boldsymbol{w}_{n}$ are reordered to $M$ antennas for OFDM modulation,

$$
\left[\boldsymbol{a}_{1} \cdots \boldsymbol{a}_{M}\right]=\left[\boldsymbol{w}_{1} \cdots \boldsymbol{w}_{N}\right]^{T},
$$

where $\boldsymbol{a}_{m} \in \mathbb{C}^{N \times 1}$ represents the frequency-domain signal to be transmitted from the $m$ th antenna. The time-domain signals are obtained through the inverse discrete Fourier transform (IDFT), i.e., $\hat{\boldsymbol{a}}_{m}=\boldsymbol{F}_{N}^{H} \boldsymbol{a}_{m}, \forall m$. Then, a cyclic prefix (CP) is added to the time-domain samples of each antenna to eliminate intersymbol interference (ISI). Finally, these samples are 


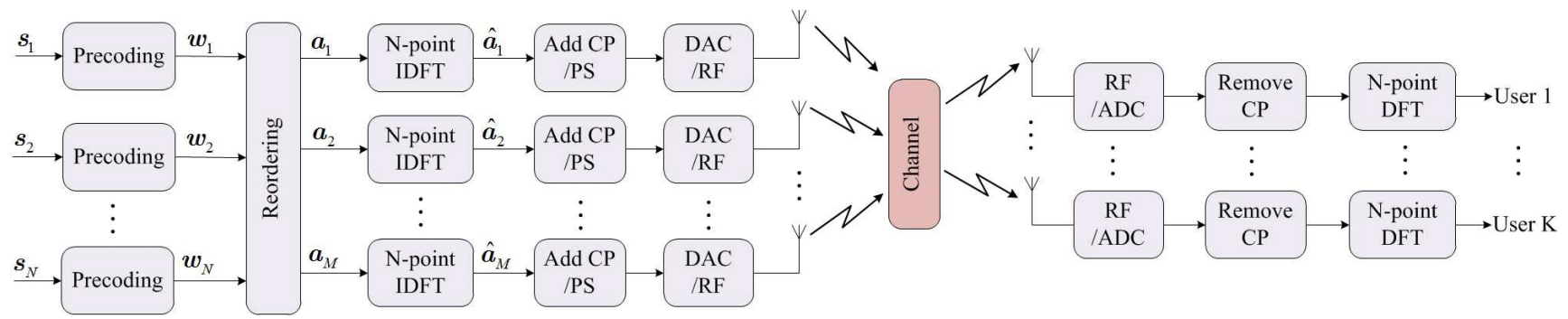

Fig. 1. System model for the downlink of OFDM based massive MIMO, with $N$ OFDM tones, $M$ transmit antennas and $K$ independent single-antenna users.

converted to analog signals and transmitted via the frequencyselective channel.

At the receivers, after removing the CPs of the received signals, the DFT is performed to obtain the frequency-domain signals. The receive vector consisting of $K$ users' signals can be described as

$$
\boldsymbol{r}_{n}=\boldsymbol{H}_{n} \boldsymbol{w}_{n}+\boldsymbol{e}_{n}, \quad \forall n
$$

where $\boldsymbol{r}_{n} \in \mathbb{C}^{K \times 1}$ denotes the receive vector associated with the $n$th tone, and $\boldsymbol{e}_{n} \in \mathbb{C}^{K \times 1}$ is the receiver noise and has i.i.d. circularly symmetric complex Gaussian entries with zeromean and variance $N_{o}$. If the ZF precoding scheme is used, by combining (1), (2) and (4), the received signal vector equals to $\boldsymbol{r}_{n}=\boldsymbol{s}_{n}+\boldsymbol{e}_{n}, \forall n$, which means the MUI is perfectly removed.

\section{B. Peak-to-Average Power Ratio (PAPR) Reduction}

OFDM modulation typically exhibits a large dynamic range because the phases of the sub-carriers are independent of each other, which may combine in a constructive or destructive manner. To avoid out-of-band radiation and signal distortion, high-solution DACs and linear power amplifiers are required at the transmitter to accommodate the large peaks of OFDM signals, which leads to expensive and power-inefficient RF chains.

PAPR is defined as the ratio of the peak power of the signal to its average power. Specifically, the PAPR at the $m$ th transmit antenna is defined as

$$
\operatorname{PAPR}_{m} \triangleq \frac{2 N\left\|\hat{\boldsymbol{a}}_{m}\right\|_{\widetilde{\infty}}^{2}}{\left\|\hat{\boldsymbol{a}}_{m}\right\|_{2}^{2}}
$$

where the operator $\|\cdot\|_{\infty}^{2}$ is used because RF-chains often process and modulate the real and imaginary part of timedomain samples independently. It should also be noted that, we only consider the PAPR of discrete-time OFDM signals in this paper, one can obtain its continuous-time counterpart precisely by implementing an $L$-times oversampling in OFDM modulation [16] 1 Since many conventional MIMO-OFDM systems, such as 3GPP LTE [17] and IEEE 802.11.n [18], disallow such an oversampling operation, here we ignore the difference as in [12] (i.e., $L=1$ ).

When the number of transmit antennas is larger than the number of users, numerous ZF precoding matrices are available. In other words, for a set of $s_{n}, n=1, \ldots, N$, we have

\footnotetext{
${ }^{1}$ Instead of $N$-point IDFT, $L$-times oversampling can be implemented by $L N$-point IDFT of the frequency-domain signals with $(L-1) N$ zero-padding.
}

an infinite number of precoded signals $\boldsymbol{w} \triangleq\left[\boldsymbol{w}_{1}^{T}, \ldots, \boldsymbol{w}_{N}^{T}\right]^{T}$ that achieve perfect MUI cancelation. Thus there may exist a candidate $\boldsymbol{w}$ whose associated time-domain signals $\left\{\hat{\boldsymbol{a}}_{m}\right\}$ have low PAPRs. In this paper, instead of designing the procoding matrix, we directly search for the signal $\boldsymbol{w}$ to achieve a joint PAPR reduction and MUI cancelation. Specifically, in order to remove the MUI, the precoded vectors $\boldsymbol{w}_{n}$ need to satisfy:

$$
\begin{array}{ll}
\boldsymbol{s}_{n}=\boldsymbol{H}_{n} \boldsymbol{w}_{n}, & n \in \mathcal{T}, \\
\mathbf{0}_{M \times 1}=\boldsymbol{w}_{n}, & n \in \mathcal{T}^{c} .
\end{array}
$$

The whole linear constraints of (6) can be further written as

$$
\bar{s}=\bar{H} w
$$

where $\bar{s} \in \mathbb{C}^{N K \times 1}$ denotes the concatenation of all vectors on the left-hand side of (6), $\overline{\boldsymbol{H}}$ is a block diagonal matrix with its diagonal blocks equal to $\boldsymbol{H}_{n}$ for $n \in \mathcal{T}$ and $\boldsymbol{I}_{M}$ for $n \in \mathcal{T}^{c}$. According to (3), the reordering operation can be equivalently written as a linear transformation, i.e.,

$$
a=T w,
$$

where $\boldsymbol{a}=\left[\boldsymbol{a}_{1}^{T}, \ldots, \boldsymbol{a}_{M}^{T}\right]^{T}, \boldsymbol{T}$ is a permutation matrix that assigns the $M$ entries of each precoded vector to the $M$ antennas respectively. Recalling $\hat{\boldsymbol{a}}_{m}=\boldsymbol{F}_{N}^{H} \boldsymbol{a}_{m}, \forall m$, (7) and (8), we have

$$
\overline{\boldsymbol{s}}=\overline{\boldsymbol{H}} \boldsymbol{T}^{T} \overline{\boldsymbol{F}} \widehat{\boldsymbol{a}},
$$

where $\overline{\boldsymbol{F}} \triangleq \boldsymbol{I}_{M} \otimes \boldsymbol{F}_{N}$, and $\widehat{\boldsymbol{a}} \triangleq\left[\hat{\boldsymbol{a}}_{1}^{T}, \ldots, \hat{\boldsymbol{a}}_{M}^{T}\right]^{T}$. Given a symbol vector $\bar{s}$, our goal is to search for a signal $\widehat{a}$ satisfying the above equation (9), and meanwhile its sub-vector $\hat{\boldsymbol{a}}_{m}$, i.e. the signal to be transmitted at each antenna, having a low PAPR. This problem can be formulated as a minimax problem which minimizes the maximum PAPR among all antennas subject to the linear constraint defined in (9). Nevertheless, this problem, as indicated in [12], is complex to solve. To circumvent the difficulty, the minimax problem is replaced by a constrained optimization which minimizes the $\ell_{\widetilde{\infty}}$ norm of $\widehat{a}$, a vector formed by aggregating all time-domain vectors $\left\{\hat{\boldsymbol{a}}_{m}\right\}$ [12]

$$
\min \|\widehat{\boldsymbol{a}}\|_{\widetilde{\infty}} \quad \text { subject to } \overline{\boldsymbol{s}}=\overline{\boldsymbol{H}} \boldsymbol{T}^{T} \overline{\boldsymbol{F}} \widehat{\boldsymbol{a}} .
$$

This problem can be further converted into a real-valued form as follows [12]

$$
\min \|\boldsymbol{x}\|_{\infty} \quad \text { subject to } \boldsymbol{y}=\boldsymbol{A} \boldsymbol{x},
$$


where

$$
\begin{gathered}
\boldsymbol{y} \triangleq\left[\begin{array}{c}
\Re\{\overline{\boldsymbol{s}}\} \\
\Im\{\overline{\boldsymbol{s}}\}
\end{array}\right], \boldsymbol{x} \triangleq\left[\begin{array}{l}
\Re\{\widehat{\boldsymbol{a}}\} \\
\Im\{\widehat{\boldsymbol{a}}\}
\end{array}\right], \\
\boldsymbol{A} \triangleq\left[\begin{array}{cc}
\Re\left\{\overline{\boldsymbol{H}} \boldsymbol{T}^{T} \overline{\boldsymbol{F}}\right\} & -\Im\left\{\overline{\boldsymbol{H}} \boldsymbol{T}^{T} \overline{\boldsymbol{F}}\right\} \\
\Im\left\{\overline{\boldsymbol{H}} \boldsymbol{T}^{T} \overline{\boldsymbol{F}}\right\} & \Re\left\{\overline{\boldsymbol{H}} \boldsymbol{T}^{T} \overline{\boldsymbol{F}}\right\}
\end{array}\right],
\end{gathered}
$$

and the dimension of $\boldsymbol{A}$ is $2\left(|\mathcal{T}| K+\left|\mathcal{T}^{c}\right| M\right) \times 2 N M$. For notational convenience, let $J \triangleq 2\left(|\mathcal{T}| K+\left|\mathcal{T}^{c}\right| M\right)$ and $I \triangleq$ $2 N M$.

Intuitively, via minimizing the largest magnitude of entries of $\boldsymbol{x}$, the PAPR associated with each transmit antenna can be reduced. This problem can be solved exactly by reformulating (11) as a linear programming problem, but is computationally prohibitive when the signal dimension is large. To develop an efficient algorithm, the equality constraint is relaxed as $\| \boldsymbol{y}-$ $\boldsymbol{A x} \|_{2} \leq \delta$ in [12]. Hence the optimization (11) can eventually be reformulated as

$$
\min \lambda\|\boldsymbol{x}\|_{\infty}+\|\boldsymbol{y}-\boldsymbol{A x}\|_{2}^{2},
$$

where $\lambda>0$ is a regularization parameter. An efficient numerical method, namely, the fast iterative truncation algorithm (FITRA), was employed [12] to solve (12). The FITRA algorithm requires to choose a suitable regularization parameter $\lambda$ to balance between the PAPR reduction and the data fitting error, which may be tricky in practice. In the following, we develop a Bayesian method which is free of this issue, and also turns out to be more efficient and effective than the FITRA algorithm.

\section{BAYESIAN MODEL}

To facilitate our algorithm development, we introduce a noise term to model the mismatch between $\boldsymbol{y}$ and $\boldsymbol{A x}$, i.e.

$$
\boldsymbol{y}=\boldsymbol{A x}+\boldsymbol{\epsilon},
$$

where $\epsilon$ denotes the noise vector and its entries are assumed to be i.i.d. Gaussian random variables with zero-mean and unknown variance $\beta^{-1}$. Here we treat $\beta$ as an unknown parameter because the Bayesian framework allows an automatic determination of its model parameters and usually provides a reasonable balance between the data fitting error and the desired characteristics of the solution. In case that there is a pre-specified tolerance value for the MUI, we can also set an appropriate value for $\beta$ instead of treating it as unknown.

To reduce the PAPR associated with each transmit antenna, we aim to find a quasi-constant magnitude solution to the above underdetermined linear system. Note that a constant magnitude signal achieves a minimum PAPR. Ideally we hope to find a solution with all of its entries having a constant magnitude. Nevertheless, it is highly unlikely that there exists such a solution to satisfy (or approximately satisfy with a tolerable error) the MUI cancelation equality, i.e. (13). Therefore we, alternatively, seek a quasi-constant magnitude solution with as many entries as possible located on the boundary points of an interval $[-v, v]$, whereas the rest entries bounded within $[-v, v]$ but not restricted to lie on the boundary points in order to meet the MUI cancelation constraint.
To encourage a quasi-constant magnitude solution, we propose a hierarchical truncated Gaussian mixture prior for the signal $\boldsymbol{x}$. In the first layer, coefficients of $\boldsymbol{x}$ are assumed independent of each other and each entry $x_{i}$ is assigned a truncated Gaussian mixture distribution:

$$
p\left(x_{i}\right)=\left\{\begin{array}{cl}
\pi \frac{\mathcal{N}\left(x_{i} ; v, \alpha_{i 1}^{-1}\right)}{\eta_{i 1}}+(1-\pi) \frac{\mathcal{N}\left(x_{i} ;-v, \alpha_{i 2}^{-1}\right)}{\eta_{i 2}} & \text { if } x_{i} \in[-v, v], \\
0 & \text { otherwise, }
\end{array}\right.
$$

where the first component of (14) is characterized by a truncated Gaussian distribution with its mean and variance given by $v$ and $\alpha_{i 1}^{-1}$, respectively; the second component is characterized by a truncated Gaussian distribution with its mean and variance given by $-v$ and $\alpha_{i 2}^{-1}$, respectively; $\pi \in[0,1]$ is a mixing coefficient that denotes the probability of generating $x_{i}$ from the first component; the distribution lies within the interval $[-v, v]$, i.e. from the mean of the second component to the mean of the first component; and $\eta_{i l}$ is a normalization constant of the $l$ th component, given by

$$
\eta_{i 1}=\frac{1}{2}-\Phi\left(-2 v \sqrt{\alpha_{i 1}}\right), \quad \eta_{i 2}=\Phi\left(2 v \sqrt{\alpha_{i 2}}\right)-\frac{1}{2} .
$$

The second layer specifies Gamma distributions as hyperpriors over the precision parameters $\boldsymbol{\alpha}_{1} \triangleq\left\{\alpha_{i 1}\right\}_{i=1}^{I}$ and $\boldsymbol{\alpha}_{2} \triangleq$ $\left\{\alpha_{i 2}\right\}_{i=1}^{I}$ :

$$
p\left(\boldsymbol{\alpha}_{1}, \boldsymbol{\alpha}_{2} ; a, b\right)=\prod_{l=1}^{2} \prod_{i=1}^{I} \operatorname{Gamma}\left(\alpha_{l i} \mid a, b\right),
$$

where

$$
\operatorname{Gamma}(\alpha \mid a, b)=\Gamma(a)^{-1} b^{a} \alpha^{a-1} e^{-b \alpha},
$$

in which $\Gamma(a)=\int_{0}^{\infty} t^{a-1} e^{-t} d t$ is the gamma function. To make the hyperpriors non-informative, small values of $a$ and $b$, e.g. $a=b=10^{-6}$, should be used [19]. Note that the choice of the Gamma hyperprior over the precision is inspired by [19]. As indicated in [19], the Gamma hyperprior with $a=$ $b=10^{-6}$ corresponds to a broad hyperprior which allows the precision (more precisely, the posterior mean of the precision) to become arbitrarily large. For our case, we also place a broad hyperprior on the precision parameters such that some of these precision parameters are allowed to become arbitrarily large. As a consequence, the corresponding entries will be driven towards and eventually located on the boundary points.

The prior distributions with different model hyperparameters $\alpha_{i 1}, \alpha_{i 2}$ are illustrated in Fig. 2, where $\pi$ and $v$ are both set to 0.5 . We can see that the prior distribution defined in (14) resembles the shape of a bowl. Thus the prior has the potential to push the entries of the solution toward its boundaries. In addition, the use of the Gamma hyperprior allows the posterior mean of the precision to become arbitrarily large. As a result, the associated entries $x_{i}$ will eventually lie on one of the two boundary points, leading to a quasi-constant magnitude solution. The graphical model of the proposed hierarchical is presented in Fig. 3 (a).

In general, Bayesian inference requires computing the logarithm of the prior. In this regard, (14) is a inconvenient form 


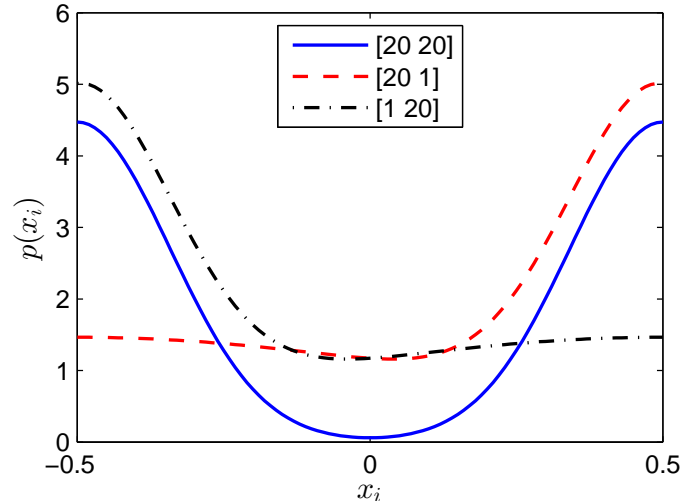

Fig. 2. Prior distribution function with different $\left[\begin{array}{ll}\alpha_{i 1} & \alpha_{i 2}\end{array}\right]$, in which $\pi$ and $v$ are both set to 0.5 .

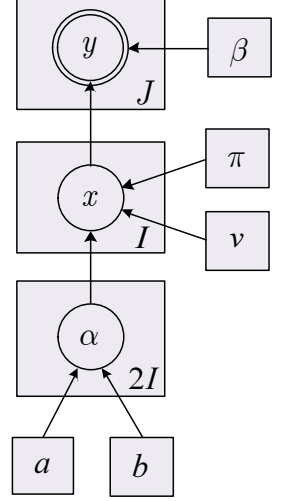

(a)

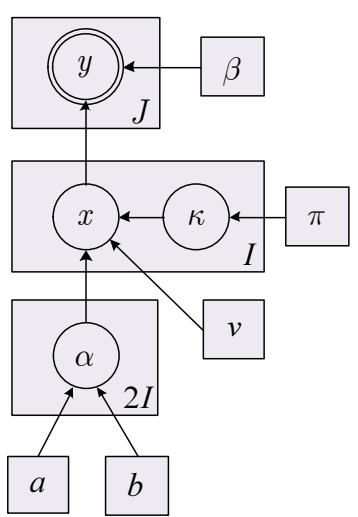

(b)
Fig. 3. Graphical models for low-PAPR signal priors, with circles denoting hidden variables, double circles denoting observed variables and squares representing model parameters. (a) Original prior, (b) Modified prior.

for inference. To address this issue, we turn the prior into an exponential form by introducing a binary latent variable $\kappa_{i}$ indicating which component is selected for $x_{i}$, i.e., $\kappa_{i}=1$ indicates the first component is selected while $\kappa_{i}=0$ corresponds to the second component. The equivalent prior can be written as

$$
\begin{aligned}
& p\left(x_{i} \mid \alpha_{i 1}, \alpha_{i 2}, \kappa_{i} ; v\right) \\
& =\left(\frac{\mathcal{N}\left(x_{i} ; v, \alpha_{i 1}^{-1}\right)}{\eta_{i 1}}\right)^{\kappa_{i}}\left(\frac{\mathcal{N}\left(x_{i} ;-v, \alpha_{i 2}^{-1}\right)}{\eta_{i 2}}\right)^{1-\kappa_{i}}, x_{i} \in[-v, v],
\end{aligned}
$$

and the distribution for $\kappa_{i}$ is

$$
p\left(\kappa_{i} ; \pi\right)=(\pi)^{\kappa_{i}}(1-\pi)^{1-\kappa_{i}}
$$

where the mixing coefficient is set to $\pi=0.5$ to make the prior non-informative. Also, we define $\boldsymbol{\kappa} \triangleq\left\{\kappa_{i}\right\}_{i=1}^{I}$. The updated graphical model is shown in Fig. 3 (b). Note that, according to (18) and (19), we can compute the conditional distribution $p\left(x_{i} \mid \alpha_{i 1}, \alpha_{i 2} ; v\right)$ via $p\left(x_{i} \mid \alpha_{i 1}, \alpha_{i 2} ; v\right)=$ $\sum_{\kappa_{i}} p\left(x_{i} \mid \alpha_{i 1}, \alpha_{i 2}, \kappa_{i} ; v\right) p\left(\kappa_{i} ; \pi\right)$, which results in the same form of (14).

\section{BAYESIAN INFERENCE}

We now proceed to perform Bayesian inference for the proposed hierarchical model. A variational expectation- maximization (EM) strategy is employed for the Bayesian inference. In our model, $\boldsymbol{z} \triangleq\left\{\boldsymbol{x}, \boldsymbol{\alpha}_{1}, \boldsymbol{\alpha}_{2}, \boldsymbol{\kappa}\right\}$ are treated as hidden variables. The noise variance $\beta$ and the boundary parameter $v$ are unknown deterministic parameters, i.e. $\boldsymbol{\theta} \triangleq\{\beta, v\}$. Before proceeding, we provide a brief review of the variational EM algorithm.

\section{A. Variational Bayesian Methodology}

Consider a probabilistic model with observed data $\boldsymbol{y}$, hidden variables $\boldsymbol{z}$ and unknown deterministic parameters $\boldsymbol{\theta}$. It is straightforward to show that the marginal probability of the observed data can be decomposed into two terms

$$
\ln p(\boldsymbol{y} ; \boldsymbol{\theta})=F(q, \boldsymbol{\theta})+\mathrm{KL}(q \| p),
$$

where

$$
F(q, \boldsymbol{\theta})=\int q(\boldsymbol{z}) \ln \left(\frac{p(\boldsymbol{y}, \boldsymbol{z} ; \boldsymbol{\theta})}{q(\boldsymbol{z})}\right) d \boldsymbol{z}
$$

and

$$
\mathrm{KL}(q \| p)=-\int q(\boldsymbol{z}) \ln \left(\frac{p(\boldsymbol{z} \mid \boldsymbol{y} ; \boldsymbol{\theta})}{q(\boldsymbol{z})}\right) d \boldsymbol{z},
$$

where $q(\boldsymbol{z})$ is any probability density function, $\operatorname{KL}(q \| p)$ is the Kullback-Leibler divergence between $p(\boldsymbol{z} \mid \boldsymbol{y} ; \boldsymbol{\theta})$ and $q(\boldsymbol{z})$. Since $\operatorname{KL}(q \| p) \geq 0$, it follows that $F(q, \boldsymbol{\theta})$ is a lower bound of $\ln p(\boldsymbol{y} ; \boldsymbol{\theta})$, with the equality holds only when $\operatorname{KL}(q \| p)=0$, which implies $p(\boldsymbol{z} \mid \boldsymbol{y} ; \boldsymbol{\theta})=q(\boldsymbol{z})$. The EM algorithm can be viewed as an iterative algorithm which iteratively maximizes the lower bound $F(q, \boldsymbol{\theta})$ with respect to the distribution $q(\boldsymbol{z})$ and the parameters $\boldsymbol{\theta}$.

Assume that the current estimate of the parameters is $\boldsymbol{\theta}^{\mathrm{OLD}}$. The EM algorithm evaluates $q^{\mathrm{NEW}}(\boldsymbol{z})$ by maximizing $F\left(q, \boldsymbol{\theta}^{\mathrm{OLD}}\right)$ with respect to $q(\boldsymbol{z})$ in the E-step, and then finds new parameter estimate $\boldsymbol{\theta}^{\mathrm{NEW}}$ by maximizing $F\left(q^{\mathrm{NEW}}, \boldsymbol{\theta}\right)$ with respect to $\boldsymbol{\theta}$ in the M-step. It is easy to see that when $q^{\mathrm{NEW}}(\boldsymbol{z})=p\left(\boldsymbol{z} \mid \boldsymbol{y} ; \boldsymbol{\theta}^{\mathrm{OLD}}\right)$, the lower bound $F\left(q, \boldsymbol{\theta}^{\mathrm{OLD}}\right)$ is maximized. Nevertheless, in practice, the posterior distribution $p\left(\boldsymbol{z} \mid \boldsymbol{y} ; \boldsymbol{\theta}^{\mathrm{OLD}}\right)$ is usually computationally intractable. To address this difficulty, we could assume $q(\boldsymbol{z})$ has some specific parameterized functional form and conduct optimization over the designated form. A particular form of $q(\boldsymbol{z})$ that has been widely used with great success is the factorized form over the component variable or the block component variable $\left\{z_{i}\right\}$ in $\boldsymbol{z}$ [20], i.e. $q(\boldsymbol{z})=\prod_{i} q_{i}\left(z_{i}\right)$. We therefore can compute the approximate posterior by finding $q(\boldsymbol{z})$ of the factorized form that maximizes the lower bound $F\left(q, \boldsymbol{\theta}^{\mathrm{OLD}}\right)$. The maximization can be conducted in an alternating fashion for each hidden variable, which leads to [20]

$$
q_{i}\left(z_{i}\right) \propto \exp \left(\langle\ln p(\boldsymbol{y}, \boldsymbol{z} ; \boldsymbol{\theta})\rangle_{k \neq i}\right) .
$$

where $\langle\cdot\rangle_{k \neq i}$ denotes an expectation with respect to the distributions $q_{k}\left(z_{k}\right)$ for all $k \neq i$.

Then in the M-step, a new estimate of $\boldsymbol{\theta}$ is obtained by maximizing the Q-function

$$
Q\left(\boldsymbol{\theta}, \boldsymbol{\theta}^{\mathrm{OLD}}\right)=\langle\ln p(\boldsymbol{y}, \boldsymbol{z} ; \boldsymbol{\theta})\rangle_{q(\boldsymbol{z})} .
$$




\section{B. Likelihood Function Approximation via GAMP}

Let $\boldsymbol{z} \triangleq\left\{\boldsymbol{x}, \boldsymbol{\alpha}_{1}, \boldsymbol{\alpha}_{2}, \boldsymbol{\kappa}\right\}$ denote all hidden variables appearing in our hierarchical model, and $\boldsymbol{\theta} \triangleq\{\beta, v\}$ denote the unknown deterministic parameters. As discussed in the previous subsection, the posterior of $\boldsymbol{z}$ can be approximated by a factorized form as follows

$$
\begin{aligned}
& p\left(\boldsymbol{x}, \boldsymbol{\alpha}_{1}, \boldsymbol{\alpha}_{2}, \boldsymbol{\kappa} \mid \boldsymbol{y} ; \beta, v\right) \\
& \quad \approx q\left(\boldsymbol{x}, \boldsymbol{\alpha}_{1}, \boldsymbol{\alpha}_{2}, \boldsymbol{\kappa}\right)=q(\boldsymbol{x}) q\left(\boldsymbol{\alpha}_{1}\right) q\left(\boldsymbol{\alpha}_{2}\right) q(\boldsymbol{\kappa}) .
\end{aligned}
$$

Following (23), the approximate posteriors can be obtained as

$$
\begin{aligned}
\ln q(\boldsymbol{x}) & =\left\langle\ln p\left(\boldsymbol{y}, \boldsymbol{x}, \boldsymbol{\alpha}_{1}, \boldsymbol{\alpha}_{2}, \boldsymbol{\kappa} ; \beta, v\right)\right\rangle_{q\left(\boldsymbol{\alpha}_{1}\right) q\left(\boldsymbol{\alpha}_{2}\right) q(\boldsymbol{\kappa})}+\mathrm{const} \\
\ln q\left(\boldsymbol{\alpha}_{1}\right) & =\left\langle\ln p\left(\boldsymbol{y}, \boldsymbol{x}, \boldsymbol{\alpha}_{1}, \boldsymbol{\alpha}_{2}, \boldsymbol{\kappa} ; \beta, v\right)\right\rangle_{q(\boldsymbol{x}) q\left(\boldsymbol{\alpha}_{2}\right) q(\boldsymbol{\kappa})}+\mathrm{const} \\
\ln q\left(\boldsymbol{\alpha}_{2}\right) & =\left\langle\ln p\left(\boldsymbol{y}, \boldsymbol{x}, \boldsymbol{\alpha}_{1}, \boldsymbol{\alpha}_{2}, \boldsymbol{\kappa} ; \beta, v\right)\right\rangle_{q(\boldsymbol{x}) q\left(\boldsymbol{\alpha}_{1}\right) q(\boldsymbol{\kappa})}+\mathrm{const} \\
\ln q(\boldsymbol{\kappa}) & =\left\langle\ln p\left(\boldsymbol{y}, \boldsymbol{x}, \boldsymbol{\alpha}_{1}, \boldsymbol{\alpha}_{2}, \boldsymbol{\kappa} ; \beta, v\right)\right\rangle_{q(\boldsymbol{x}) q\left(\boldsymbol{\alpha}_{1}\right) q\left(\boldsymbol{\alpha}_{2}\right)}+\mathrm{const}
\end{aligned}
$$

We first consider the calculation of $q(\boldsymbol{x})$. Keeping those terms that are dependent on $\boldsymbol{x}$, we have

$$
\begin{aligned}
& \ln q(\boldsymbol{x}) \\
& =\left\langle\ln p(\boldsymbol{y} \mid \boldsymbol{x} ; \beta) p\left(\boldsymbol{x} \mid \boldsymbol{\alpha}_{1}, \boldsymbol{\alpha}_{2}, \boldsymbol{\kappa} ; v\right)\right\rangle_{q\left(\boldsymbol{\alpha}_{1}\right) q\left(\boldsymbol{\alpha}_{2}\right) q(\boldsymbol{\kappa})}+\mathrm{const} \\
& =\frac{1}{2} \sum_{i=1}^{I}\left\langle-\alpha_{i 1} \kappa_{i}\left(x_{i}-v\right)^{2}-\alpha_{i 2}\left(1-\kappa_{i}\right)\left(x_{i}+v\right)^{2}\right\rangle \\
& \quad+\ln p(\boldsymbol{y} \mid \boldsymbol{x} ; \beta)+\mathrm{const} \quad \text { if } x_{i} \in[-v, v] \forall i, \quad \text { (27) }
\end{aligned}
$$

and $\ln q(\boldsymbol{x})=-\infty$ otherwise, where the subscripts of $\langle\cdot\rangle_{q(\cdot)}$ are omitted for simplicity. Since the variables $\left\{x_{i}\right\}$ in the joint likelihood function $p(\boldsymbol{y} \mid \boldsymbol{x} ; \beta)$ are non-factorizable, obtaining the posterior $q(\boldsymbol{x})$ is rather difficult. To overcome this difficulty, we employ the generalized approximate message passing (GAMP) technique [14] to obtain an amiable approximation of the joint likelihood function $p(\boldsymbol{y} \mid \boldsymbol{x} ; \beta)$.

GAMP is a simplification of loopy BP, and can be used to compute approximate marginal posteriors and likelihoods. Here we approximate the joint likelihood function $p(\boldsymbol{y} \mid \boldsymbol{x} ; \beta)$ as a product of approximate marginal likelihoods computed via the GAMP, i.e.

$$
p(\boldsymbol{y} \mid \boldsymbol{x} ; \beta) \approx \hat{p}(\boldsymbol{y} \mid \boldsymbol{x} ; \beta) \propto \prod_{i=1}^{I} \mathcal{N}\left(x_{i} \mid \hat{r}_{i}, \tau_{i}^{r}\right),
$$

where $\mathcal{N}\left(x_{i} \mid \hat{r}_{i}, \tau_{i}^{r}\right)$ is the approximate marginal likelihood obtained by the GAMP algorithm. To calculate $\hat{r}_{i}$ and $\tau_{i}^{r}$, an estimate of the posterior $q(\boldsymbol{x})$ and $\beta$ is required as inputs to the GAMP algorithm (see the details of the GAMP algorithm provided below). Hence the GAMP algorithm can be embedded in the variational EM framework: given an estimate of $q(\boldsymbol{x})$ and $\beta$, use the GAMP to obtain an approximation of the likelihood function $p(\boldsymbol{y} \mid \boldsymbol{x} ; \beta)$; with the approximation $\hat{p}(\boldsymbol{y} \mid \boldsymbol{x} ; \beta)$, the variational EM proceeds to yield a new estimate of $q(\boldsymbol{x})$ and $\beta$, along with estimates of other deterministic parameters (e.g. $v$ ) and posterior distributions for the other hidden variables (e.g. $\left.\boldsymbol{\alpha}_{1}, \boldsymbol{\alpha}_{2}, \boldsymbol{\kappa}\right)$. This iterative procedure is illustrated in Fig. 4 .

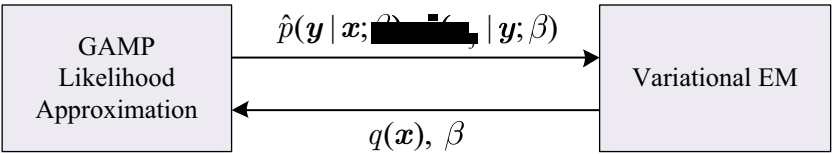

Fig. 4. Proposed variational EM-GAMP framework, where the hatted distribution $\hat{p}(\cdot)$ represents an approximation of $p(\cdot)$.

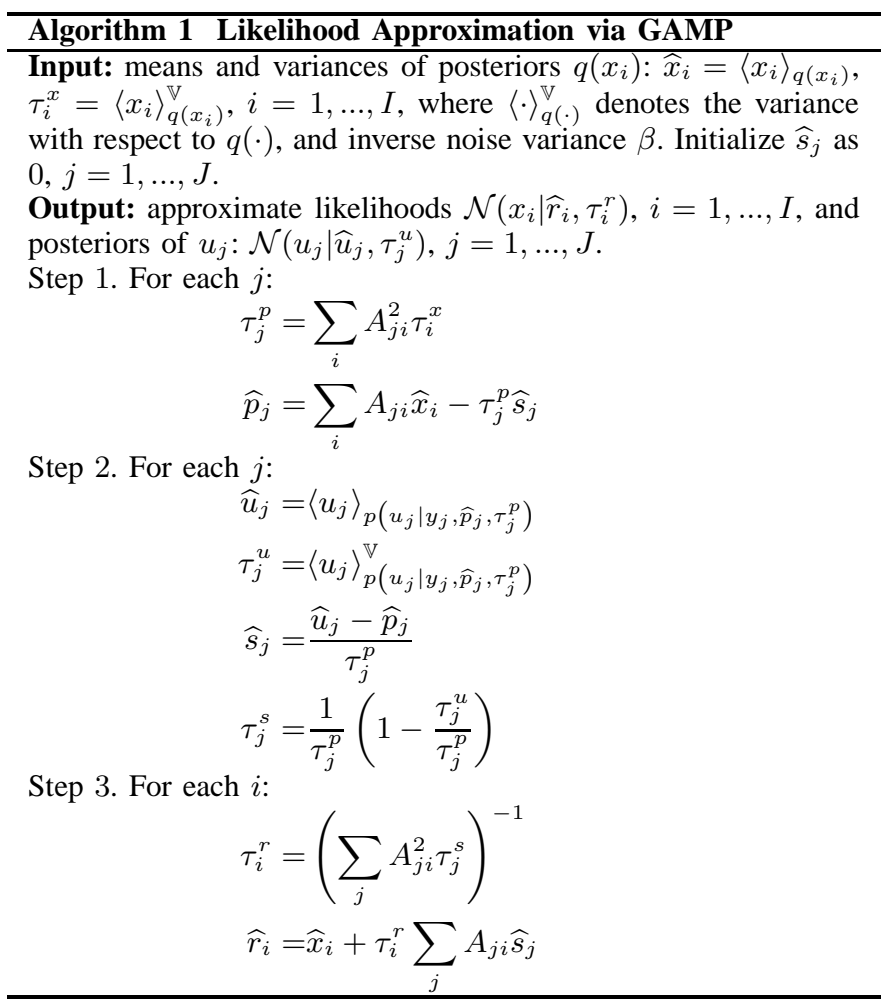

Note that besides the approximation $\hat{p}\left(\boldsymbol{y} \mid x_{i} ; \beta\right)$, GAMP also produces approximations for the marginal posteriors of the noiseless output $\boldsymbol{u}=\left[u_{1}, \ldots, u_{J}\right]^{T} \triangleq \boldsymbol{A x}$, which are given by

$$
\begin{aligned}
p\left(u_{j} \mid \boldsymbol{y}, \beta\right) & \approx \hat{p}\left(u_{j} \mid \boldsymbol{y}, \beta\right) \\
& \propto p\left(y_{j} \mid u_{j} ; \beta\right) \mathcal{N}\left(u_{j} \mid \hat{p}_{j}, \tau_{j}^{p}\right),
\end{aligned}
$$

where $\hat{p}_{j}$ and $\tau_{j}^{p}$ are quantities obtained from the GAMP algorithm. Since the noise is assumed to be white Gaussian noise, we have $\hat{p}\left(u_{j} \mid \boldsymbol{y}, \beta\right)=\mathcal{N}\left(u_{j} \mid \widehat{u}_{j}, \tau_{j}^{z}\right)$, where

$$
\tau_{j}^{u}=\frac{\tau_{j}^{p}}{\tau_{j}^{p} \beta+1} \quad \widehat{u}_{j}=\tau_{j}^{u}\left(y_{j} \beta+\frac{\widehat{p}_{j}}{\tau_{j}^{p}}\right) .
$$

As will be shown later, this approximation can be used to learn the inverse of the noise variance, $\beta$, in the M-step.

Remarks: Generalized approximate message passing (GAMP) is a very-low-complexity Bayesian iterative technique recently developed in [14] for obtaining approximate marginal posteriors and likelihoods. It therefore can be naturally embedded within the EM framework to provide an approximate posterior distribution of $\boldsymbol{x}$ and reduce the computational complexity, as shown in [21], [22]. Specifically, the EM-GAMP framework of [21], [22] proceeds in a doubleloop manner: the outer loop (EM) computes the Q-function using the approximate posterior distribution of $\boldsymbol{x}$, and 
maximizes the Q-function to update the model parameters (e.g. $\left.\boldsymbol{\alpha}_{1}, \boldsymbol{\alpha}_{2}, \boldsymbol{\kappa}\right)$; the inner loop (GAMP) utilizes the newly estimated parameters to obtain a new approximation of the posterior distribution of $\boldsymbol{x}$. However, this procedure is not suitable for our variational EM framework, because from the GAMP's point of view, the hyperparameters $\left\{\boldsymbol{\alpha}_{1}, \boldsymbol{\alpha}_{2}, \boldsymbol{\kappa}\right\}$ need to be known and fixed in order to compute an approximate posterior distribution of $\boldsymbol{x}$, while the variational EM treats the model parameters (e.g. $\boldsymbol{\alpha}_{1}, \boldsymbol{\alpha}_{2}, \boldsymbol{\kappa}$ ) as latent variables. Therefore, instead of computing the approximate posterior distribution of $\boldsymbol{x}$, in our variational EM framework, the GAMP is simply used to obtain an amiable approximation of the likelihood function $p(\boldsymbol{y} \mid \boldsymbol{x} ; \beta)$, and this approximation involves no latent variables $\left\{\boldsymbol{\alpha}_{1}, \boldsymbol{\alpha}_{2}, \boldsymbol{\kappa}\right\}$. Besides, unlike the EM-GAMP framework where the inner loop (GAMP) is implemented in an iterative way, in our proposed variational EM-GAMP framework, as detailed in Algorithm 1, the GAMP only needs to go through one iteration to obtain an approximation of the likelihood function. In fact, the GAMP algorithm described here is a simplified version of the original GAMP algorithm by retaining only its first three steps and skipping its iterative procedure. Note that the original GAMP algorithm involves a four-step iterative process, in which the fourth step computes the posterior of $\boldsymbol{x}$ by using the approximate likelihood function obtained from the first three steps.

Note that we can also treat $\left\{\boldsymbol{\alpha}_{1}, \boldsymbol{\alpha}_{2}, \boldsymbol{\kappa}\right\}$ as deterministic parameters and resort to the EM-GAMP framework for Bayesian inference. Nevertheless, in this case, we need to estimate a set of binary parameters $\left\{\kappa_{i}\right\}$ in the M-step. This is essentially a combinatorial search problem and the binary estimation may cause the algorithm to get stuck in undesirable local minima.

GAMP is known to work well for $\boldsymbol{A}$ with i.i.d zero-mean sub-Gaussian entries, but may fail for a rank-deficient $\boldsymbol{A}$. One may refer to the method [23] to improve the stability of the GAMP against the ill-condition of the matrix $\boldsymbol{A}$. Nevertheless, GAMP is expected to perform well in wireless communication scenarios since indoor and urban outdoor environments are typically rich in scattering and entries of MIMO channel matrices are usually assumed to be i.i.d Gaussian [15], [24].

\section{E-Step: Update of Hidden Variables}

Update of $q(\boldsymbol{x})$ : As discussed above, $p(\boldsymbol{y} \mid \boldsymbol{x} ; \beta)$ is approximated as a factorized form of $I$ independent scalar likelihoods, which enables the computation of $q(\boldsymbol{x})$ (27). Specifically, using (28), 27) can be simplified as

$$
\begin{aligned}
& \ln q(\boldsymbol{x}) \\
& =\frac{1}{2} \sum_{i=1}^{I}\left\langle-\alpha_{i 1} \kappa_{i}\left(x_{i}-v\right)^{2}-\alpha_{i 2}\left(1-\kappa_{i}\right)\left(x_{i}+v\right)^{2}\right\rangle \\
& \quad-\frac{1}{2} \sum_{i=1}^{I}\left(x_{i}-\widehat{r}_{i}\right)^{2} / \tau_{i}^{r}+\mathrm{const} \\
& =-\sum_{i=1}^{I}\left(\frac{1}{2}\left(\left\langle\kappa_{i}\right\rangle\left\langle\alpha_{i 1}\right\rangle-\left\langle\kappa_{i}\right\rangle\left\langle\alpha_{i 2}\right\rangle+\left\langle\alpha_{i 2}\right\rangle+1 / \tau_{i}^{r}\right) x_{i}^{2}\right. \\
& \left.\quad+\left(\left(\left\langle\kappa_{i}\right\rangle\left\langle\alpha_{i 1}\right\rangle+\left\langle\kappa_{i}\right\rangle\left\langle\alpha_{i 2}\right\rangle-\left\langle\alpha_{i 2}\right\rangle\right) v+\widehat{r}_{i} / \tau_{i}^{r}\right) x_{i}\right)+ \text { const } \\
& \quad \text { if } x_{i} \in[-v, v] \forall i,
\end{aligned}
$$

and $\ln q(\boldsymbol{x})=-\infty$ otherwise. It can be seen that $\ln q(\boldsymbol{x})$ has a factorized form, which implies that hidden variables $\left\{x_{i}\right\}$ have independent posterior distributions. Also, it can be readily verified that the posterior $q\left(x_{i}\right)$ follows a truncated Gaussian distribution

$$
q\left(x_{i}\right)=\left\{\begin{array}{cl}
\frac{\mathcal{N}\left(x_{i} \mid \mu_{i}, \sigma_{i}^{2}\right)}{\phi_{i}} & \text { if } x_{i} \in[-v, v], \\
0 & \text { otherwise }
\end{array}\right.
$$

where the variance $\sigma_{i}^{2}$, mean $\mu_{i}$ and the normalization constant $\phi_{i}$ are given respectively as

$$
\begin{aligned}
\sigma_{i}^{2} & =\left(\left\langle\kappa_{i}\right\rangle\left\langle\alpha_{i 1}\right\rangle-\left\langle\kappa_{i}\right\rangle\left\langle\alpha_{i 2}\right\rangle+\left\langle\alpha_{i 2}\right\rangle+1 / \tau_{i}^{r}\right)^{-1}, \\
\mu_{i} & =\left(\left(\left\langle\kappa_{i}\right\rangle\left\langle\alpha_{i 1}\right\rangle+\left\langle\kappa_{i}\right\rangle\left\langle\alpha_{i 2}\right\rangle-\left\langle\alpha_{i 2}\right\rangle\right) v+\widehat{r}_{i} / \tau_{i}^{r}\right) \sigma_{i}^{2}, \\
\phi_{i} & =\Phi\left(\left(v-\mu_{i}\right) / \sigma_{i}\right)-\Phi\left(\left(-v-\mu_{i}\right) / \sigma_{i}\right) .
\end{aligned}
$$

Update of $q\left(\boldsymbol{\alpha}_{1}\right)$ : Keeping only the terms that depend on $\boldsymbol{\alpha}_{1}$, the variational optimization of $q\left(\boldsymbol{\alpha}_{1}\right)$ yields

$$
\begin{aligned}
& \ln q\left(\boldsymbol{\alpha}_{1}\right) \\
& =\left\langle\ln p\left(\boldsymbol{x} \mid \boldsymbol{\alpha}_{1}, \boldsymbol{\alpha}_{2}, \boldsymbol{\kappa} ; v\right) p\left(\boldsymbol{\alpha}_{1}\right)\right\rangle_{q(\boldsymbol{x}) q\left(\boldsymbol{\alpha}_{2}\right) q(\boldsymbol{\kappa})}+\mathrm{const} \\
& =\sum_{i=1}^{I}\left\langle\ln p\left(x_{i} \mid \alpha_{i 1}, \alpha_{i 2}, \kappa_{i} ; v\right) p\left(\alpha_{i 1}\right)\right\rangle_{q(\boldsymbol{x}) q\left(\boldsymbol{\alpha}_{2}\right) q(\boldsymbol{\kappa})}+\mathrm{const} \\
& =-\sum_{i=1}^{I}\left\langle\kappa_{i}\right\rangle \ln \eta_{i 1}+\sum_{i=1}^{I}\left(\left(a+\frac{1}{2}\left\langle\kappa_{i}\right\rangle-1\right) \ln \alpha_{i 1}\right. \\
& \left.\quad-\left(b+\frac{1}{2}\left\langle\kappa_{i}\right\rangle\left\langle\left(x_{i}-v\right)^{2}\right\rangle\right) \alpha_{i 1}\right)+ \text { const. }
\end{aligned}
$$

We see that $\ln q\left(\boldsymbol{\alpha}_{1}\right)$ also has a factorized form $\ln q\left(\boldsymbol{\alpha}_{1}\right)=$ $\sum_{i} \ln q\left(\alpha_{i 1}\right)$. Note that $\eta_{i 1}$ (defined in (15)) is a function of $\alpha_{i 1}$, which makes the inference of $q\left(\alpha_{i 1}\right)$ difficult. To address this difficulty, we use the latest computed value to replace $\eta_{i 1}$ i.e. let $\ln \eta_{i 1} \approx \ln \eta_{i 1}^{(t)}$. Note that similar approximations were also adopted in [22] to facilitate the inference. With this approximation, we obtain

$$
\begin{aligned}
& \ln q\left(\alpha_{i 1}\right) \\
& =\left(a+\frac{1}{2}\left\langle\kappa_{i}\right\rangle-1\right) \ln \alpha_{i 1}-\left(b+\frac{1}{2}\left\langle\kappa_{i}\right\rangle\left\langle\left(x_{i}-v\right)^{2}\right\rangle\right) \alpha_{i 1} \\
& \quad+\text { const. }
\end{aligned}
$$


Therefore $q\left(\alpha_{i 1}\right)$ follows a Gamma distribution

$$
q\left(\alpha_{i 1}\right)=\operatorname{Gamma}\left(\alpha_{i 1} \mid \widetilde{a}_{i 1}, \widetilde{b}_{i 1}\right)
$$

with

$$
\begin{aligned}
& \widetilde{a}_{i 1}=a+\frac{1}{2}\left\langle\kappa_{i}\right\rangle \\
& \widetilde{b}_{i 1}=b+\frac{1}{2}\left\langle\kappa_{i}\right\rangle\left\langle\left(x_{i}-v\right)^{2}\right\rangle .
\end{aligned}
$$

Update of $q\left(\boldsymbol{\alpha}_{2}\right)$ : Following a procedure similar to the derivation of $q\left(\boldsymbol{\alpha}_{1}\right)$, we have

$$
q\left(\alpha_{i 2}\right)=\operatorname{Gamma}\left(\alpha_{i 2} \mid \widetilde{a}_{i 2}, \widetilde{b}_{i 2}\right)
$$

with

$$
\begin{aligned}
& \widetilde{a}_{i 2}=a+\frac{1}{2}\left(1-\left\langle\kappa_{i}\right\rangle\right) \\
& \widetilde{b}_{i 2}=b+\frac{1}{2}\left(1-\left\langle\kappa_{i}\right\rangle\right)\left\langle\left(x_{i}+v\right)^{2}\right\rangle .
\end{aligned}
$$

Update of $q(\boldsymbol{\kappa})$ : The approximate posterior distribution $q_{\kappa}(\boldsymbol{\kappa})$ can be computed as

$$
\begin{aligned}
& \ln q(\boldsymbol{\kappa}) \\
& =\left\langle\ln p\left(\boldsymbol{x} \mid \boldsymbol{\alpha}_{1}, \boldsymbol{\alpha}_{2}, \boldsymbol{\kappa} ; v\right) p(\boldsymbol{\kappa})\right\rangle_{q(\boldsymbol{x}) q\left(\boldsymbol{\alpha}_{1}\right) q\left(\boldsymbol{\alpha}_{2}\right)}+\mathrm{const} \\
& =\sum_{i=1}^{I}\left\langle\ln p\left(x_{i} \mid \alpha_{i 1}, \alpha_{i 2}, \kappa_{i} ; v\right) p\left(\kappa_{i}\right)\right\rangle_{q\left(x_{i}\right) q\left(\alpha_{i 1}\right) q\left(\alpha_{i 2}\right)}+\mathrm{const} \\
& =\sum_{i=1}^{I}\left(\frac{1}{2}\left(\left\langle\ln \alpha_{i 1}\right\rangle-\left\langle\ln \alpha_{i 2}\right\rangle-\left\langle\left(x_{i}-v\right)^{2}\right\rangle+\left\langle\left(x_{i}+v\right)^{2}\right\rangle\right)\right. \\
& \left.\quad+\left\langle\ln \eta_{i 2}\right\rangle-\left\langle\ln \eta_{i 1}\right\rangle+\ln \frac{\pi}{1-\pi}\right) \kappa_{i}+\text { const. }
\end{aligned}
$$

We see that $\ln q(\boldsymbol{\kappa})=\sum_{i} \ln q\left(\kappa_{i}\right)$ and, moreover, the posterior $q\left(\kappa_{i}\right)$ obeys a Bernoulli distribution, i.e. $\kappa_{i}$ takes values zero or one, and the corresponding probability can be computed from (44). To simplify computation, we can use the approximation $\left\langle\ln \eta_{i l}\right\rangle \approx \ln \eta_{i l}^{(t)}, l=1,2$.

In summary, the variational Bayesian inference involves updates of the approximate posterior distributions for hidden variables $\boldsymbol{x}, \boldsymbol{\alpha}_{1}, \boldsymbol{\alpha}_{2}$ and $\boldsymbol{\kappa}$ in an alternating fashion. Some of the expectations and moments used during the update are summarized as

$$
\begin{aligned}
& \left\langle x_{i}\right\rangle=\mu_{i}-\frac{\sigma_{i}^{2}}{\phi_{i}}\left(\mathcal{N}\left(v \mid \mu_{i}, \sigma_{i}^{2}\right)-\mathcal{N}\left(-v \mid \mu_{i}, \sigma_{i}^{2}\right)\right) \\
& \left\langle x_{i}^{2}\right\rangle=u_{i}\left\langle x_{i}\right\rangle+\sigma_{i}^{2}-\frac{\sigma_{i}^{2}}{\phi_{i}}\left(\mathcal{N}\left(v \mid \mu_{i}, \sigma_{i}^{2}\right)+\mathcal{N}\left(-v \mid \mu_{i}, \sigma_{i}^{2}\right)\right) \\
& \left\langle\alpha_{i l}\right\rangle=\widetilde{a}_{i l} / \widetilde{b}_{i l}, \quad l=1,2 \\
& \left\langle\ln \alpha_{i l}\right\rangle=\psi\left(\widetilde{a}_{i l}\right)-\ln \widetilde{b}_{i l}, \quad l=1,2 \\
& \left\langle\kappa_{i}\right\rangle=q\left(\kappa_{i}=1\right)
\end{aligned}
$$

where

$$
\psi(a) \triangleq \frac{\partial \ln \Gamma(a)}{\partial a}
$$

is known as the digamma function [25].
Discussions: We can gain some insight into our proposed algorithm by examining the update rules for precision parameters $\left\{\alpha_{i 1}, \alpha_{i 2}\right\}$. Since $a$ and $b$ are set very small, the update rules (47) for $\left\{\alpha_{i 1}, \alpha_{i 2}\right\}$ are approximately given by

$$
\begin{aligned}
\left\langle\alpha_{i 1}\right\rangle & =\frac{1}{\left\langle\left(x_{i}-v\right)^{2}\right\rangle} \\
\left\langle\alpha_{i 2}\right\rangle & =\frac{1}{\left\langle\left(x_{i}+v\right)^{2}\right\rangle}
\end{aligned}
$$

We see that the posterior mean of the precision, say $\left\langle\alpha_{i 1}\right\rangle$, is inversely proportional to the distance between the entry and the boundary point $v$. When $x_{i}$ is close to the boundary point $v$, the posterior mean of the precision $\alpha_{i 1}$ will become large. As a consequence, the prior distribution becomes sharp around the boundary point $v$. Hence the prior has the potential to push the entry $x_{i}$ closer to the boundary point $v$, which in turn results in a larger $\left\langle\alpha_{i 1}\right\rangle$ according to (47). This feedback mechanism keeps pushing most of the entries towards the boundary until they are eventually located on one of the boundary points. Our simulation results further corroborate our above discussions: the proposed algorithm yields a solution with a substantial percentage of entries lying exactly on the boundary points.

\section{M-Step: Update of Deterministic Parameters}

As indicated earlier, in the variational EM framework, the deterministic parameters $\boldsymbol{\theta}=\{\beta, v\}$ are estimated by maximizing the $\mathrm{Q}$-function, i.e.

$$
\boldsymbol{\theta}^{\mathrm{NEW}}=\max _{\boldsymbol{\theta}} Q\left(\boldsymbol{\theta}, \boldsymbol{\theta}^{\mathrm{OLD}}\right)=\langle\ln p(\boldsymbol{y}, \boldsymbol{z} ; \boldsymbol{\theta})\rangle_{q(\boldsymbol{z})} .
$$

Update of $\beta$ : We fist discuss the update of the parameter $\beta$, the inverse of the noise variance. Since the GAMP algorithm provides an approximate posterior distribution for the noiseless output $\boldsymbol{u} \triangleq \boldsymbol{A x}$, we can simply treat $\boldsymbol{u}$ as hidden variables when computing the Q-function, i.e.

$$
\begin{aligned}
Q\left(\beta, \beta^{(t)}\right) & =\sum_{j=1}^{J}\left\langle\ln p\left(y_{j} \mid u_{j} ; \beta\right)\right\rangle_{\hat{p}\left(u_{j} \mid \boldsymbol{y}, \beta\right)}+\mathrm{const} \\
& =\frac{J}{2} \ln \beta-\frac{1}{2} \beta \sum_{j=1}^{J}\left\langle\left(y_{j}-u_{j}\right)^{2}\right\rangle+\text { const. }
\end{aligned}
$$

The new estimate of $\beta$ is obtained by maximizing the Qfunction, which can be solved by setting the derivative of $Q\left(\beta, \beta^{(t)}\right)$ with respect to $\beta$ to zero. The derivative is given as

$$
\frac{\partial Q\left(\beta, \beta^{(t)}\right)}{\partial \beta}=\frac{J}{2 \beta}-\frac{1}{2} \sum_{j=1}^{J}\left\langle\left(y_{j}-u_{j}\right)^{2}\right\rangle .
$$

Setting it to zero, we obtain

$$
\beta^{(t+1)}=\frac{J}{\sum_{j=1}^{J}\left\langle\left(y_{j}-u_{j}\right)^{2}\right\rangle} .
$$

Update of $v$ : We now discuss how to update the boundary parameter $v$. The boundary parameter $v$ can be updated by maximizing the Q-function with respect to $v$. Nevertheless, the optimization is complex since the Q-function involves computing the expectation of the normalization terms $\eta_{i l}$, 
$i=1, \ldots, I, l=1,2$, with respect to the posterior distributions $p\left(\alpha_{i l}\right)$. Here we propose a heuristic approach to update $v$. The basic idea is to find an appropriate value of $v$ such that the mismatch $\|\boldsymbol{y}-\boldsymbol{A} \hat{\boldsymbol{x}}\|_{2}^{2}$ is minimized, where $\hat{\boldsymbol{x}}$ denotes the estimated signal which is chosen as the mean of the posterior distribution $q(\boldsymbol{x})$. Note that when the boundary parameter $v$ is small, the mismatch could be large since there may not exist a solution to satisfy the constraint $\boldsymbol{y}=\boldsymbol{A} \boldsymbol{x}$ given that $\|\boldsymbol{x}\|_{\infty} \leq v$. Therefore we can firstly set a small value of $v$, then gradually increase $v$ by a step-size such that the mismatch keeps decreasing and eventually becomes negligible. Define $\delta(\hat{\boldsymbol{x}}) \triangleq\|\boldsymbol{y}-\boldsymbol{A} \hat{\boldsymbol{x}}\|_{2}^{2}$. Specifically, the step-size $\Delta v$ can be obtained by solving the following optimization problem:

$$
\Delta v=\min _{\Delta v} \delta\left(\hat{\boldsymbol{x}}^{(t)}+\gamma \Delta v\right)
$$

where $\hat{\boldsymbol{x}}^{(t)}$ denotes the estimate (i.e. posterior mean of $q(\boldsymbol{x})$ ) of the signal at iteration $t$, and $\gamma \triangleq\left[\gamma_{1}, \ldots, \gamma_{I}\right]^{T}$ is defined as

$$
\gamma_{i}=\left\{\begin{array}{rl}
1, & \text { if } \hat{x}_{i}^{(t)} \geq 0 \\
-1, & \text { if } \hat{x}_{i}^{(t)}<0
\end{array} .\right.
$$

The rationale behind the optimization (57) can be explained as follows. Since our proposed algorithm yields a solution with most of its entries located on the boundary points, if we increase the boundary $v$ by a sufficiently small step-size $\Delta v$, we can expect that the signal $\boldsymbol{x}$ will expand accordingly. We wish to find a step-size $\Delta v$ such that the expanded signal will result in a reduced mismatch. The problem (57) is a scalar least-square problem, and its solution is given by

$$
\Delta v=\frac{\left(\boldsymbol{y}-\boldsymbol{A} \hat{\boldsymbol{x}}^{(t)}\right)^{T} \boldsymbol{A} \boldsymbol{\gamma}}{\|\boldsymbol{A} \gamma\|_{2}^{2}}
$$

Then $v$ can be updated as

$$
v^{(t+1)}=v^{(t)}+\Delta v
$$

\section{E. Summary}

In summary, our algorithm is developed by resorting to the variational EM strategy. The GAMP technique is embedded in the variational EM framework to obtain an approximation of the joint likelihood function $p(\boldsymbol{y} \mid \boldsymbol{x}, \beta)$ which has a factorized form in terms of the variables $\left\{x_{i}\right\}$. Specifically, the algorithm involves an iterative process as follows: given an estimate of $q(\boldsymbol{x})$ and $\beta$, we use the GAMP to obtain an approximation of the likelihood function $p(\boldsymbol{y} \mid \boldsymbol{x} ; \beta)$; with the approximation $\hat{p}(\boldsymbol{y} \mid \boldsymbol{x} ; \beta)$, the variational EM proceeds to yield a new estimate of $q(\boldsymbol{x})$ and $\beta$, along with the approximate posteriors of the other hidden variables and an estimate of the boundary parameter $v$. For clarity, we summarize our proposed algorithm as follows.

Note that the dominating operations of the proposed algorithm in each iteration only involve simple matrix-vector multiplications, which scales as $\mathcal{O}(J I)(J<I)$. Thus the proposed algorithm has a computational complexity comparable to the FITRA algorithm [12] which also has a computational complexity of $\mathcal{O}(J I)$ per iteration. Besides, as will be shown in our experiments, the proposed algorithm has a much faster convergence rate than the FITRA algorithm, which is more

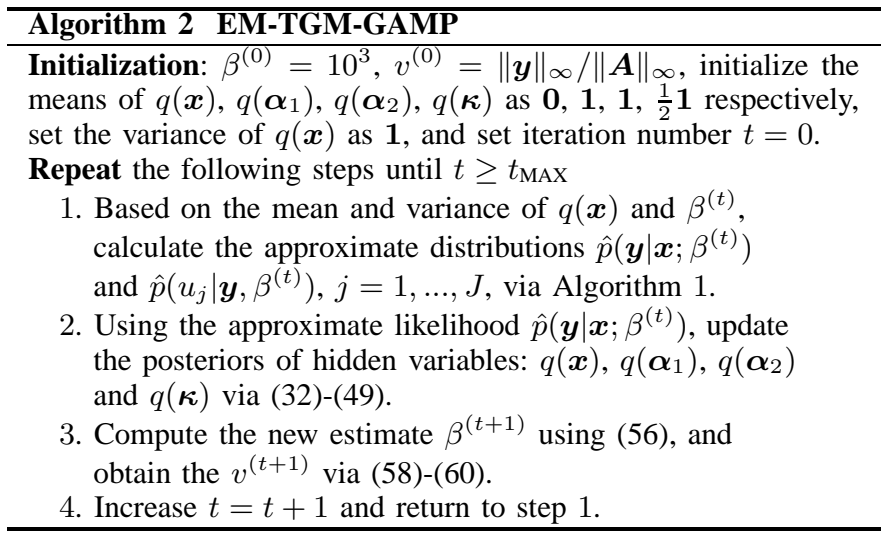

favorable for real-time implementation needed for practical systems.

\section{Simulation Results}

We now carry out experiments to illustrate the effectiveness of the proposed truncated Gaussian mixture (TGM) modelbased variational EM-GAMP algorithm 2 (referred to as the EM-TGM-GAMP). We compare our approach with the FITRA algorithm [12], the zero-forcing (ZF) precoding scheme, and the amplitude clipping scheme [4] in which the $\mathrm{ZF}$ is first employed and then the peaks of the resulting signal are clipped with a specified threshold.

In our simulations, we consider a MIMO system which has $M=100$ antennas at the BS and serves $K=10$ singleantenna users. A 16-QAM constellation is considered, and the number of OFDM tones is set to $N=128$, in which only $|\mathcal{T}|=114$ tones are used for data transmission [18]. We assume that the channel is frequency-selective and modeled as a tap-delay line with $D=8$ taps. The time-domain channel response matrices $\widehat{\boldsymbol{H}}_{d}, d=1, \ldots, D$, have i.i.d. circularly symmetric Gaussian distributed entries with zero mean and unit variance. The frequency-domain response matrix $\boldsymbol{H}_{n}$ can be obtained as

$$
\boldsymbol{H}_{n}=\sum_{d=1}^{D} \widehat{\boldsymbol{H}}_{d} \exp \left(\frac{-j 2 \pi d n}{N}\right) .
$$

For the FITRA algorithm, the regularization parameter is set to be $\lambda=0.25$ as suggested by [12]. Also, unless explicitly stated otherwise, the maximum number of iterations of the FITRA and the EM-TGM-GAMP are set to be 2000 and 200, respectively.

The complementary cumulative distribution function (CCDF) is used to evaluate the PAPR reduction performance. The CCDF denotes the probability that the PAPR of the estimated signal exceeds a given threshold $\mathrm{PAPR}_{0}$, i.e.

$$
\operatorname{CCDF}\left(\mathrm{PAPR}_{0}\right)=\operatorname{Pr}\left(\mathrm{PAPR}>\mathrm{PAPR}_{0}\right) .
$$

Also, to evaluate the multiuser interference of the transmit signals, we define the MUI as

$$
\text { MUI }=\frac{\sum_{n \in \mathcal{T}}\left\|\boldsymbol{s}_{n}-\boldsymbol{H}_{n} \boldsymbol{w}_{n}\right\|_{2}^{2}}{\sum_{n \in \mathcal{T}}\left\|\boldsymbol{s}_{n}\right\|_{2}^{2}}
$$

\footnotetext{
${ }^{2}$ Codes are available at http://www.junfang-uestc.net/codes/EM-TGM-GAMP.rar
} 


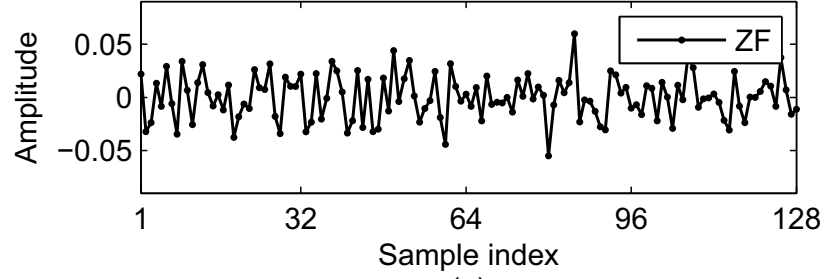

(a)

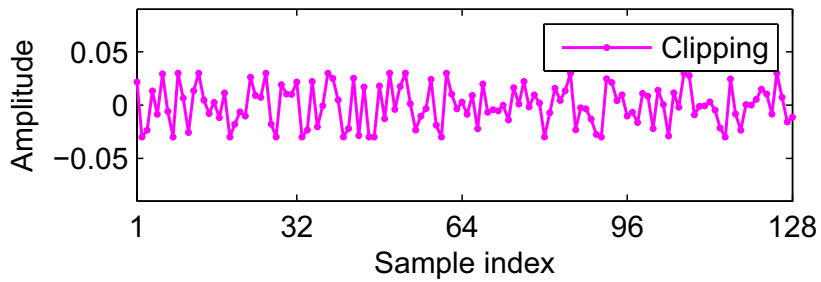

(c)

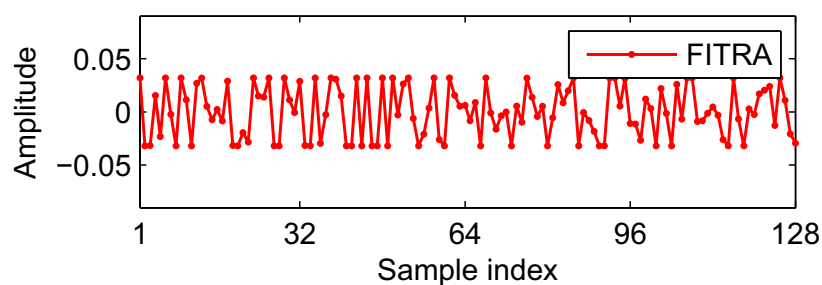

(e)

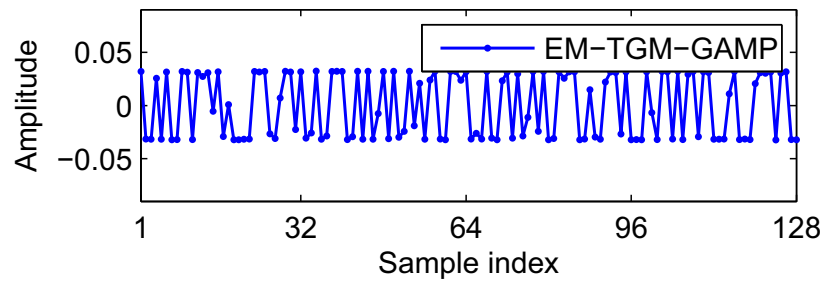

(g)

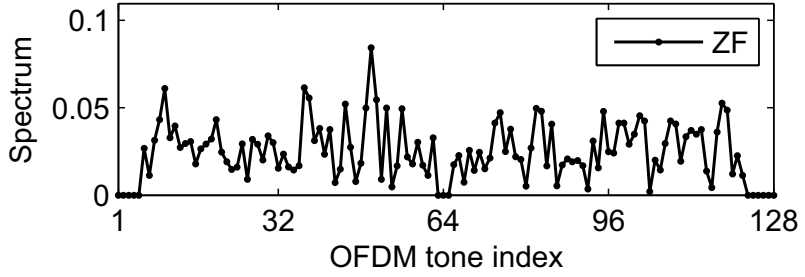

(b)

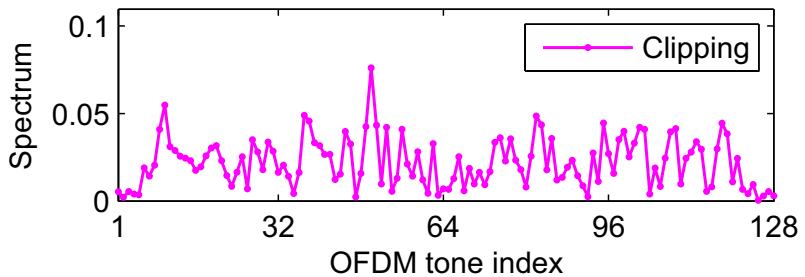

(d)

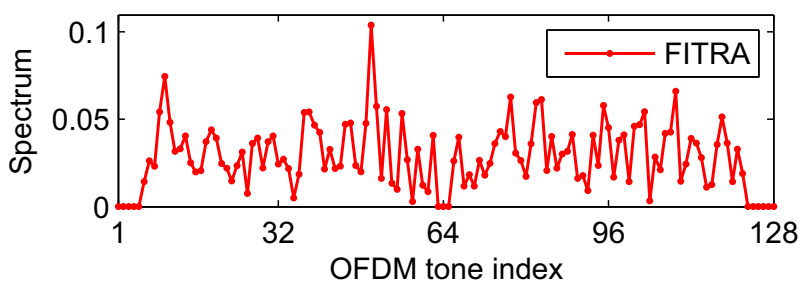

(f)

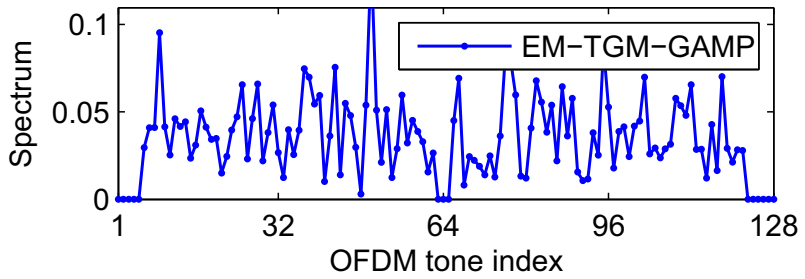

(h)

Fig. 5. Time/Frequency representation for different schemes. (a), (c), (e) and (g) are time-domain signals for ZF, clipping, FITRA and EM-TGM-GAMP, respectively (PAPR: $\mathrm{ZF}=10.6 \mathrm{~dB}$, Clipping $=4.3 \mathrm{~dB}$, FITRA $=2.4 \mathrm{~dB}$, and EM-TGM-GAMP $=0.8 \mathrm{~dB}$ ). (b), (d), (f) and (h) are frequency-domain signals for respective schemes $(\mathrm{MUI}: \mathrm{ZF}=-\infty \mathrm{dB}$, Clipping $=-15.3 \mathrm{~dB}$, FITRA $=-64.1 \mathrm{~dB}$, and EM-TGM-GAMP $=73.6 \mathrm{~dB}$; OBR: ZF $=-\infty \mathrm{dB}$, Clipping $=-13.8 \mathrm{~dB}$, FITRA $=-60 \mathrm{~dB}$, and EM-TGM-GAMP $=-70.5 \mathrm{~dB})$

Besides, the out-of-band (power) ratio (OBR) is introduced to measure the out-of-band radiation of the solution, which is define as

$$
\mathrm{OBR}=\frac{|\mathcal{T}| \sum_{n \in \mathcal{T}^{c}}\left\|\boldsymbol{w}_{n}\right\|_{2}^{2}}{\left|\mathcal{T}^{c}\right| \sum_{n \in \mathcal{T}}\left\|\boldsymbol{w}_{n}\right\|_{2}^{2}} .
$$

Note that, for the $\mathrm{ZF}$ procoding scheme, we have $\mathrm{OBR}=0$ and $\mathrm{MUI}=0$, while for the other three schemes, we always have $\mathrm{OBR}>0$ and $\mathrm{MUI}>0$.

It is interesting to examine the signals estimated by respective schemes. In the (a), (c), (e) and (g) of Fig. 5, we depict the real-part of the first transmit antenna's time-domain signal (i.e. $\hat{\boldsymbol{a}}_{1}$ ) estimated by respective schemes (the imaginary part behaves similarly). We observe that our proposed algorithm yields a solution with most of its entries (about $84.4 \%$ ) located on the boundary points, which corroborates our previous claim that the proposed truncated hierarchical Gaussian mixture model encourages a quasi-constant magnitude solution. Such a solution, clearly, has a low PAPR as it looks like a constantmodulus signal. The solution of the FITRA algorithm has fewer entries (about 49.2\%) located on the boundary points.
For the ZF scheme, its solution exhibits a large variation with a few high peaks. The solution of the clipping scheme is only a slightly alleviated version of the ZF solution. Numerical results also verify our observations: our proposed algorithm has the lowest PAPR (PAPR associated with the first transmit antenna) of $0.8 \mathrm{~dB}$, the FITRA algorithm and the clipping scheme have higher PAPRs of $2.4 \mathrm{~dB}$ and $4.3 \mathrm{~dB}$, repectively, while the ZF scheme has the highest PAPR of $10.6 \mathrm{~dB}$. We see that our proposed algorithm renders a much lower PAPR than the other three schemes. The (b), (d), (f) and (h) of Fig. 5 depict the magnitudes of the frequency-domain signal $\boldsymbol{a}_{1}$ vs. the OFDM tone index. Both the EM-TGM-GAMP and the FITRA have small MUIs and out-of-band radiations: their MUIs are given by $-73.6 \mathrm{~dB}$ and $-64.1 \mathrm{~dB}$, respectively, and OBRs are given by $-70.5 \mathrm{~dB}$ and $-60 \mathrm{~dB}$, respectively. In contrast, the clipping scheme incurs a much higher MUI and out-of-band distortion, with its MUI and OBR given by $-15.3 \mathrm{~dB}$ and $-13.8 \mathrm{~dB}$, respectively.

To better evaluate the PAPR reduction performance, we plot the CCDF of the PAPR for respective schemes in Fig. 6(a). 


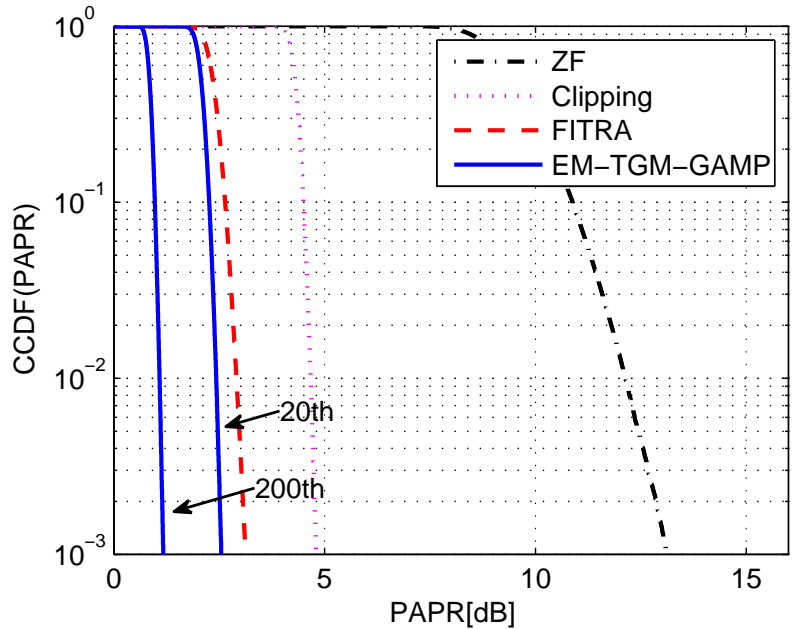

(a)

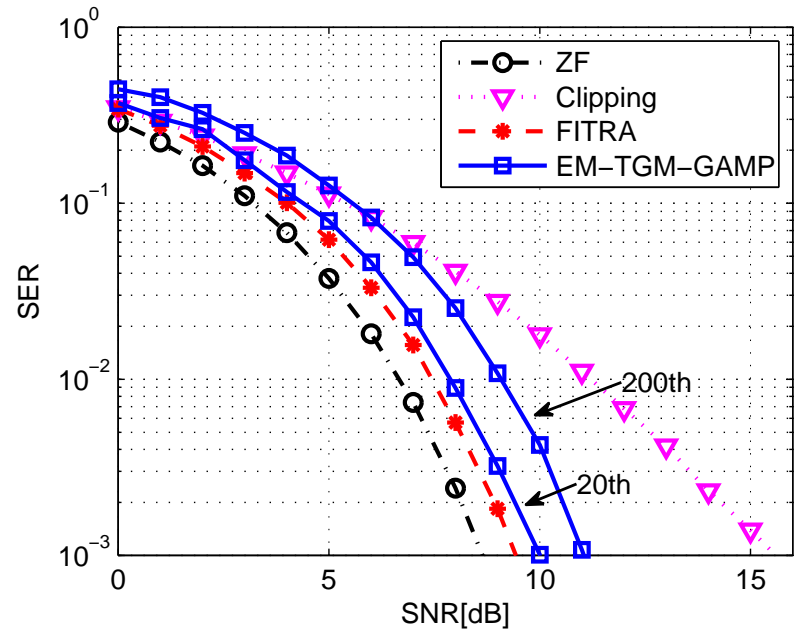

(b)

Fig. 6. PAPR and symbol error rate (SER) performance for various schemes. (a) CCDF of the PAPR, (b) SER performance.

The number of trials is chosen to be 1000 in our experiments. Note that PAPRs associated with all $M$ antennas are taken in account in calculating the empirical CCDF. We also include the results of our proposed algorithm obtained at the 20th iteration. We can see that our proposed algorithm with 200 iterations achieves a substantial PAPR reduction: it reduces the PAPR by more than $11 \mathrm{~dB}$ compared to the ZF scheme (at $\operatorname{CCDF}($ PAPR $)=1 \%$ ), by about $2 \mathrm{~dB}$ compared to the FITRA algorithm with 2000 iterations, and by about $3.2 \mathrm{~dB}$ compared to the clipping scheme. Also note that the proposed algorithm with only 20 iterations can obtain a PAPR that even is lower than the FITRA, meanwhile exhibiting a decent MUI and OBR (here MUI and OBR are averaged over 1000 independent runs) given by $-41.8 \mathrm{~dB}$ and $-21.7 \mathrm{~dB}$, respectively.

The SER performance of respective schemes is shown in Fig. 6 b), where the signal-to-noise ratio (SNR) is defined as $\mathrm{SNR}=\|\boldsymbol{x}\|_{2}^{2} / M N_{o}, N_{o}$ denotes the variance of the receiver noise (c.f. (44). We observe that the proposed algorithm incurs an SNR-performance loss of $2.5 \mathrm{~dB}$ and $1.7 \mathrm{~dB}$ (at SER = $10^{-3}$ ) compared to the ZF and FITRA schemes, respectively. This performance loss, as discussed in [12], is primarily due to an increase in the norm of the obtained solution $\boldsymbol{x}$, i.e. $\|\boldsymbol{x}\|_{2}^{2}$. It is not difficult to see that the solution obtained by our proposed method has a larger norm than the solution of the FITRA since our solution has more entries located on the boundary points. Also note that the ZF scheme renders the least-norm solution. In order to maintain the same SNR, our solution requires a stronger normalization, which causes the SER performance loss compared to the ZF and FITRA schemes. It can also be observed that the SER performance gap can be reduced if we only perform 20 iterations for our proposed method, in which case the resulting solution has fewer entries located on the boundary points and hence the increase of the norm of the solution is not that significant. Note that the performance loss of the clipping scheme is mainly caused by the residual MUI.

We now examine the convergence rates of our proposed method and the FITRA algorithm. The (a), (b) and (c) of Fig. 7 show the PAPR, MUI and OBR vs. the number of iterations, respectively. Results are averaged over 1000 inde-

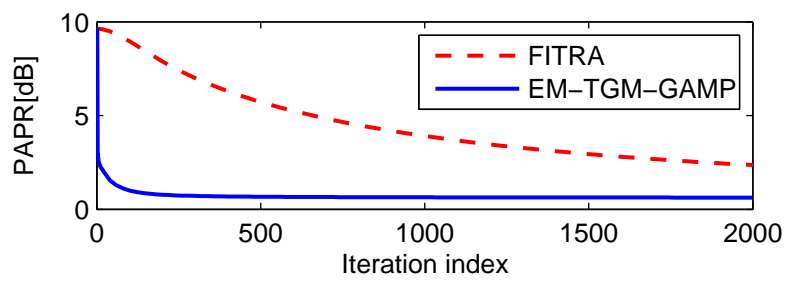

(a)

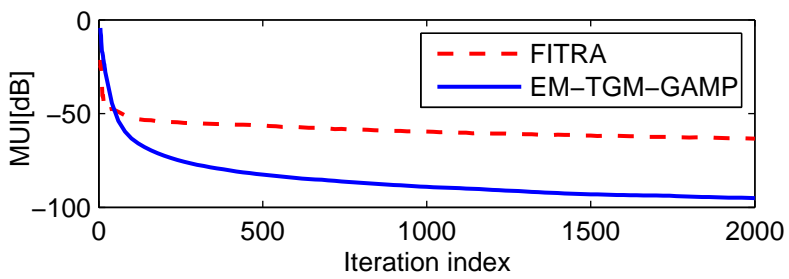

(b)

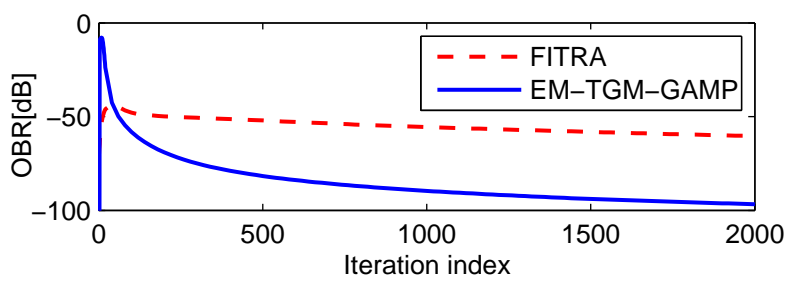

(c)

Fig. 7. Convergence rates of different metrics for EM-TGM-GAMP and FITRA. (a) PAPR, (b) MUI, (c) OBR

pendent runs and the PAPR results are averaged over PAPRs associated with all transmit antennas. Our numerical results show that the average MUI and OBR of our proposed method at the 200th iteration are $-72.5 \mathrm{~dB}$ and $-69.1 \mathrm{~dB}$ respectively, while the average MUI and OBR of the FITRA algorithm at the 2000th iteration are $-63.3 \mathrm{~dB}$ and $-60.3 \mathrm{~dB}$. With less than 200 iterations, our proposed algorithm achieves better MUI cancelation than the FITRA algorithm with even 2000th iterations. From Fig. 7 ( a), we also notice that our proposed algorithm has a fast convergence rate and is able to obtain a low-PAPR solution within only 200 iterations, whereas it 


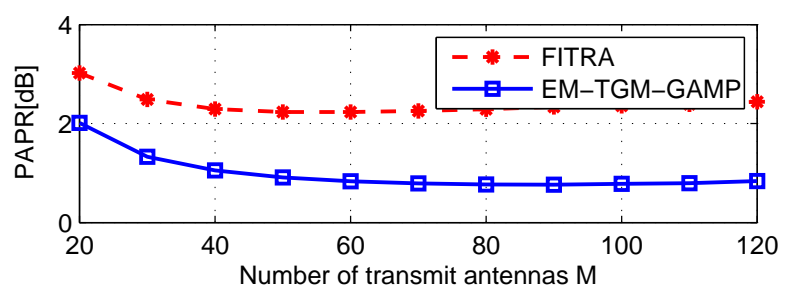

(a)

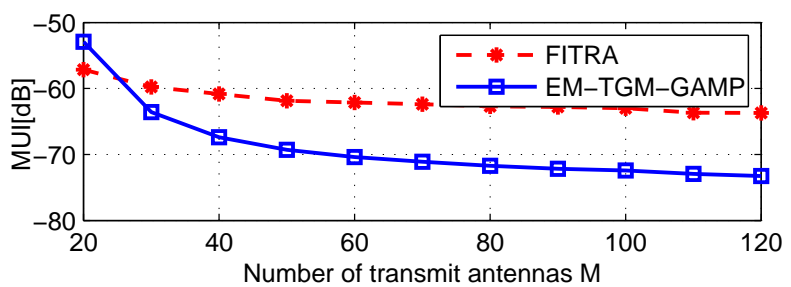

(b)

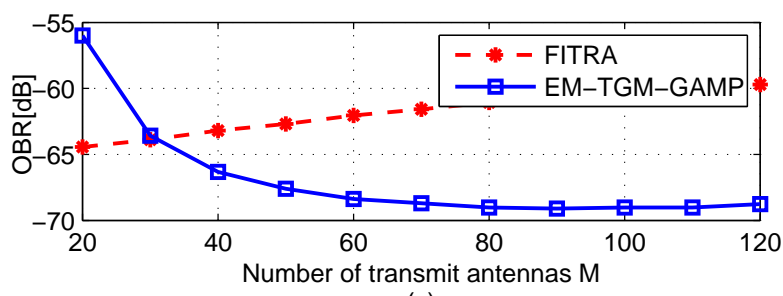

(c)

Fig. 8. (a) PAPR vs. number of transmit antennas, (b) MUI vs. number of transmit antennas, (c) OBR vs. number of transmit antennas.

takes the FITRA algorithm about 2000 iterations to achieve a reasonably low PAPR.

Lastly, we investigate the PAPR-reduction performance under different number of transmit antennas, where the number of users is fixed to be $K=10$, and the number of transmit antennas at the BS varies from 20 to 120 . Fig. 8 plots the PAPR, MUI and OBR as the number of transmit antennas varies, where results are averaged over 1000 independent runs and the PAPR results are averaged over PAPRs associated with all transmit antennas. We observe that both algorithms achieve a low PAPR when sufficient DoFs at the base station are available. Nevertheless, the proposed method is capable of exploiting the available DoFs more efficiently as the number of of transmit antennas increases.

\section{Vi. CONCLusions}

We considered the problem of joint PAPR reduction and multiuser interference (MUI) cancelation in OFDM based massive MIMO downlink systems. A hierarchical truncated Gaussian mixture prior model was proposed to encourage a low PAPR solution/signal. A variational EM algorithm was developed to obtain estimates of the hyperparameters associated with the prior model, as well as the signal. Specifically, the GAMP technique was embedded into the variational EM framework to facilitate the algorithm development. The proposed algorithm only involves simple matrixvector multiplications at each iteration, and thus has a low computational complexity. Simulation results show that the proposed algorithm achieves notable improvement in PAPR reduction as compared with the FITRA algorithm [12], and meanwhile renders better MUI cancelation and lower out-ofband radiation. The proposed algorithm also demonstrates a fast convergence rate, which makes it attractive for practical real-time systems.

\section{REFERENCES}

[1] F. Rusek, D. Persson, B. K. Lau, E. G. Larsson, T. L. Marzetta, O. Edfors, and F. Tufvesson, "Scaling up MIMO: Opportunities and challenges with verylarge arrays," IEEE Signal Process. Mag., vol. 30, no. 1, pp. 40-60, Jan. 2013.

[2] G. Wunder, R. F. Fischer, H. Boche, S. Litsyn, and J. No, "The PAPR problem in OFDM transmission: New directions for a long-lasting problem," IEEE Signal Process. Mag., vol. 30, no. 6, pp. 130-144, Jan. 2014.

[3] T. Jiang and $\mathrm{Y}$. Wu, "An overview: Peak-to-average power ratio reduction techniques for OFDM signals," IEEE Trans. Broadcasting, vol. 54, no. 2, pp. 257-268, Jun. 2008.

[4] R. ONeill and L. B. Lopes, "Envelope variations and spectral splatter in clipped multicarrier signals," in Proc. IEEE PIMRC'95, Toronto, Canada, Sep. 1995, pp. 71-75.

[5] J. Tellado, "Peak to average power reduction for multicarrier modulation," PhD thesis (Stanford University, 2000).

[6] B. S. Krongold and D. L. Jones, "PAR reduction in OFDM via active constellation extension," IEEE Trans. Broadcasting, vol. 49, no. 3, pp. 258-268, Sep. 2003.

[7] R. W. Bäuml, R. F. Fischer, and J. B. Huber, "Reducing the peak-toaverage power ratio of multicarrier modulation by selected mapping," IEE Elec. Letters, vol. 32, no. 22, pp. 2056-2057, Oct. 1996.

[8] S. H. Müller and J. B. Huber, "OFDM with reduced peak-to-average power ratio by optimum combination of partial transmit sequences," IEE Elec. Letters, vol. 33, no. 5, pp. 368-369, Feb. 1997.

[9] S. H. Han and J. H. Lee, "An overview of peak-to-average power ratio reduction techniques for multicarrier transmission," IEEE Wireless Commun., vol. 12, no. 2, pp. 56-65, Apr. 2005.

[10] R. F. H. Fischer and M. Hoch, "Directed selected mapping for peakto-average power ratio reduction in MIMO OFDM," IEE Elec. Letters, vol. 42, no. 2, pp. 1289-1290, Oct. 2006.

[11] T. Tsiligkaridis and D. L. Jones, "PAPR reduction performance by active constellation extension for diversity MIMO-OFDM systems," $J$. Electrical and Computer Eng., Sep. 2010.

[12] C. Studer and E. G. Larsson, "PAR-aware large-scale multi-user MIMOOFDM downlink," IEEE J. Sel. Areas Commun., vol. 31, no. 2, pp. 303-313, Feb. 2013.

[13] H. Prabhu, O. Edfors, J. Rodrigues, L. Liu, and F. Rusek, "A lowcomplex peak-to-average power reduction scheme for OFDM based massive MIMO systems," in Communications, Control and Signal Processing (ISCCSP), 2014 6th International Symposium on, Athens, Greece, 2014.

[14] S. Rangan, "Generalized approximate message passing for estimation with random linear mixing," IEEE International Symposium, Information Theory Proceedings (ISIT), Full version at arXiv:1010.5141, pp. 21682172, Aug. 2011.

[15] J. Chen, C. Wang, K. Wong, and C. Wen, "Low-complexity precoding design for massive multiuser MIMO systems using approximate message passing," IEEE Trans. Vehicular Technology, vol. PP, no. 99, pp. 1-8, Jul. 2015.

[16] C. Tellambura, "Computation of the continuous-time PAR of an OFDM signal with BPSK subcarriers," IEEE Commun. Lett., vol. 5, no. 5, pp. 185-187, May 2001.

[17] "3rd Generation Partnership Project; Technical Specification Group Radio Access Network; Evolved Universal Terrestrial Radio Access (EUTRA); Multiplexing and channel coding (Release 9)," 3GPP Organizational Partners TS 36.212, 2008.

[18] "IEEE Draft Standard; Part 11: Wireless LAN Medium Access Control (MAC) and Physical Layer (PHY) specifications; Amendment 4: Enhancements for Higher Throughput," P802.11n/D3.0, 2007.

[19] M. Tipping, "Sparse Bayesian learning and the relevance vector machine," Journal of Machine Learning Research, vol. 1, pp. 211-244, 2001.

[20] D. G. Tzikas, A. C. Likas, and N. P. Galatsanos, "The variational approximation for Bayesian inference," IEEE Signal Process. Mag., vol. 25, no. 6, pp. 131-146, Jan. 2008.

[21] J. Vila and P. Schniter, "Expectation-maximization Gaussian-mixture approximate message passing," IEEE Trans. Signal Process., vol. 61, no. 19 , pp. 4658-4672, Oct 2013. 
[22] — "An empirical-Bayes approach to recovering linearly constrained non-negative sparse signals," IEEE Trans. Signal Process., vol. 62, no. 18, pp. 4689-4703, Sep 2014.

[23] J. Vila, P. Schniter, S. Rangant, F. Krzakala, and L. Zdeborovd, "Adaptive damping and mean removal for the generalized approximate message passing algorithm," in IEEE International Conference on Acoustics, Speech, and Signal Processing (ICASSP), South Brisbane, QLD, pp. 2021-2025, Apr. 19-24, 2015.

[24] Q. Guo, D. Huang, S. Nordholm, J. Xi, , and Y. Yu, "Iterative frequency domain equalization with generalized approximate message passing," IEEE Signal Process. Lett., vol. 20, no. 6, pp. 559-562, Jun. 2013.

[25] M. Abramowitz and I. A. Stegun, Handbook of mathematical functions: with formulas, graphs, and mathematical tables. New York: Dover Publication, 1965. 